\title{
Comparative Analysis of Protein Expression Concomitant with DNA Methyltransferase 3A Depletion in a Melanoma Cell Line
}

\author{
Shengnan Tang ${ }^{1, \#}$, Xiaoyan Liu ${ }^{1, \#}$, Tonghua $\mathrm{Li}^{1 *}$, Haoyue Wang ${ }^{2}$, Jiangming Sun ${ }^{1}$, \\ Qian Qiao ${ }^{1}$, Jun $\mathbf{Y a o}^{3}$, Jian Fei ${ }^{2}$ \\ ${ }^{1}$ Department of Chemistry, Tongji University, Shanghai, China \\ ${ }^{2}$ School of Life Science \& Technology, Tongji University, Shanghai, China \\ ${ }^{3}$ School of Medicine, Fudan University, Shanghai, China \\ E-mail: "lith@tongji.edu.cn \\ Received March 17, 2011; revised May 3, 2011; accepted June 1, 2011
}

\begin{abstract}
DNA methyltransferase 3A (Dnmt3a), a de novo methyltransferase, has attracted a great deal of attention for its important role played in tumorigenesis. We have previously demonstrated that melanoma is unable to grow in-vivo in conditions of Dnmt3a depletion in a mouse model. In this study, we cultured the Dnmt3a depletion B16 melanoma (Dnmt3a-D) cell line to conduct a comparative analysis of protein expression con-comitant with Dnmt3a depletion in a melanoma cell line. After two-dimensional separation, by gel electro-phoresis and liquid chromatography, combined with mass spectrometry analysis (1DE-LC-MS/MS), the re-sults demonstrated that 467 proteins were up-regulated and 535 proteins were down-regulated in the Dnmt3a-D cell line compared to the negative control (NC) cell line. The Genome Ontology (GO) and KEGG pathway were used to further analyze the altered proteins. KEGG pathway analysis indicated that the MAPK signaling pathway exhibited a greater alteration in proteins, an interesting finding due to the close relation-ship with tumorigenesis. The results strongly suggested that Dnmt3a potentially controls the process of tu-morigenesis through the regulation of the proteins (JNK1, p38 $\alpha$, ERK1, ERK2, and BRAF) involved in tu-mor-related pathways, such as the MAPK signaling pathway and melanoma pathway.
\end{abstract}

Keywords: Dnmt3a, Melanoma Cell Line, 1DE-LC-MS/MS; MAPK Signaling Pathway, Melanoma Pathway

\section{Introduction}

Malignant melanoma, one of the most aggressive of all skin cancers, exhibits a high skin cancer mortality rate [1]. Upon metastasis, the disease is incurable in most affected people as melanoma does not respond to most systemic treatments and chemotherapy drugs [2].

It is well established that malignant melanoma contains at least 50 genes that exhibit differential expression as abnormal methylation changes emerge in the promoter region [3-5], and that DNA methylation patterns are established and maintained by the coordinated action of three DNA methyltransferases (Dnmts). It has been demonstrated that Dnmt1 [6,7], Dnmt3a, and Dnmt3b [8] are over-expressed in many malignant tumors [9]. Dnmt3a plays an important role in epigenetic modification that

${ }^{\#}$ These authors contributed equally to this work. has attracted a great deal of attention in recent years $[10,11]$. It has been reported that epigenetic modification is induced by hepatitis $\mathrm{B}$ virus $\mathrm{X}$ protein via interaction with de novo DNA methyltransferase-Dnmt3a [10]. Additionally, Dnmt3a has been found to maintain DNA methylation and regulate synaptic function in adult forebrain neurons [12]. Furthermore, it has been demonstrated that Dnmt3a-dependent non-promoter DNA methylation facilitates the transcription of neurogenic genes [13]. Recently, studies of Dnmt3a have focused on its effect on proliferation and apoptosis of hepatocellular carcinoma, colorectal cancer and malignant melanoma [14-16] In a previous study we demonstrated that tumor growth inhibition was mediated by Dnmt3a depletion [17].

In this study we describe the proteomic experiment and comparative analysis of protein expression in the 
Dnmt3a depletion B16 melanoma (Dnmt3a-D) cell line. The mouse B16 melanoma cell line used in our study exhibited a specific down-regulation of Dnmt3a via stable transfection using a Dnmt3a-RNAi construct. We obtained the negative control (NC) cell line through use of an unrelated, non-target, shRNA expression vector. Proteins of the NC cell line and Dnmt3a-D cell line were initially separated by one-dimensional gel electrophoresis (1DE), and the two gel tracks were subsequently split into 20 proteome fractions, respectively, and digested by trypsin. The peptide fractions were analyzed by capillary liquid chromatography and high accuracy mass spectrometric acquisition on a LTQ-Orbitrap (Thermo Scientific Germany) in the MS/MS mode using various and complementary fragmentation modes. The results of the comparative proteomics demonstrated that Dnmt3a depletion affects a large number of proteins. The Genome Ontology and KEGG pathway were used to group these altered proteins according to respective cellular components, molecular functions, and pathway. The suppressors of skin tumour development JNK1 and p38 $\alpha$ [18] were found to be up-regulated in the MAPK signaling pathway of the Dnmt3a-D cell line. The oncogene BRAF was down-regulated in the melanoma pathway of the Dnmt3a-D cell line. These results strongly suggested that Dnmt3a depletion potentially inhibits melanoma tumorigenesis by regulating the proteins involved in tumorrelated pathways.

\section{Materials and Methods}

\subsection{Cell Culture}

The B16 cells were purchased from the American Type Culture Collection (Manassas, VA), cultured in DMEM supplemented with $10 \% \mathrm{FBS}$, and maintained in a humidified incubator at $37^{\circ} \mathrm{C}$ and $5 \% \mathrm{CO}_{2}$. The sequence of Dnmt3a shRNA was $5^{\prime}$ gtgcagaaacatcgaggacTTCAAGAGAgtcctcgatgtttctg- cac 3'. A non-target shRNA with the sequence 5'gcaagtctaaccaacgcgt TTCAAGAGAacgcgttggtt - agacttgc $3^{\prime}$ was used as negative control (NC). The process of small hairpin RNA (shRNA) RNAi was performed as previously described [17]. At this point in the procedure we chose the B16 cells with Dnmt3a depletion (Dnmt3a-D) for further experiments.

\subsection{Protein Extraction and Measurement}

After harvesting the cells, the following experimental process was conducted (Figure 1). The cells were washed 2-3 times in ice-cold phosphate-buffered saline (PBS), lysed in $900 \mu \mathrm{L}$ RIPA lysis buffer (Bi Yun Tian Biotechnology Research Institute, China) plus $10 \mu \mathrm{L} 100$ $\mathrm{mM}$ phenylmethanesulfonyl fluoride (PMSF) for ap- proximately 10 - $20 \mathrm{~min}$. The samples were incubated for $2 \mathrm{~s}$ in an ice bath and exposed to an ultrasonic power of less than $70 \mathrm{~W}$, and then centrifuged for $45 \mathrm{~min}$ at $15,000 \mathrm{rpm}$ at $4^{\circ} \mathrm{C}$. The supernatants were then collected into eppendorf tubes for the next measurement.

The total protein of the cells was measured using the BCA Protein Assay (Bi Yun Tian Biotechnology Research Institute, China) with bovine serum albumin as a standard recommended by the manufacturer. The protein was stored at $-80^{\circ} \mathrm{C}$ until performance of isoelectro- focusing (IEF).

\subsection{1-DE and In-Gel Tryptic Digestion}

In order to conduct proteomic analysis on proteins expressed at a low level, shotgun proteomics based on 1-DE separation of total protein was evaluated in addition to the analysis of peptide mixtures produced by tryptic digestion of proteins in gel fragments by LC-MS/MS. Equal amounts of NC cell protein and Dnmt3a-D cell protein $(40 \mu \mathrm{g})$ were prepared in an identical manner. Respective samples were separated using small analytical immobilized $\mathrm{pH}$ gradient (IPG) strips $(7 \mathrm{~cm}, 3-10$ $\mathrm{pH}$ gradient; Bio-Rad). The proteins were electro-focused by initially using a voltage of $8 \mathrm{~V} / \mathrm{cm}$ for the stacking gel, and subsequently increasing the voltage to $15 \mathrm{~V} / \mathrm{cm}$ for the separating gel.

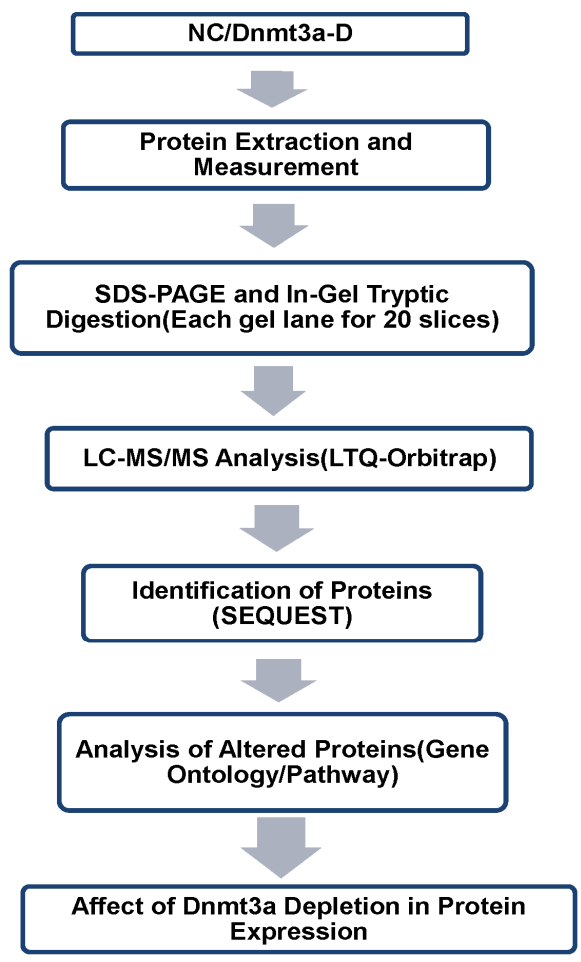

Figure 1. The experimental process of proteomic methods used in this study. 
Gel lanes were excised from the SDS-PAGE gels using a razor blade and were divided into 20 slices according to the distribution of protein (Figure 2). The proteins were separated by $12 \%$ resolving gel. Each slice was then further divided into approximately $1 \mathrm{~mm}^{3}$ pieces and placed into an eppendorf tube. The proteins were deoxidized, alkylated, dehydrated and digested. The peptide mixtures were extracted with frequent vortexing at $37^{\circ} \mathrm{C}$ for $30 \mathrm{~min}$. Samples were evaporated to dryness and stored at $-20^{\circ} \mathrm{C}$ until MS analysis.

\subsection{LC-MS/MS Analysis}

In recent years, the high performance and sensitivity of the linear quadrupole ion trap-orbitrap (LTQ-Orbitrap) mass spectrometer has interested researchers due to the capacity for top-down analysis of complete protein from tissue, body fluid, and cells [19,20].

The sample was separated using online reverse-phase nanoscale capillary liquid chromatography, and then analyzed by electrospray tandem mass spectrometry. The peptide mixtures loaded and desalted on a C18 trap column (0.5 mm diameter, $2 \mathrm{~mm}$, MICHROM,USA) using a Tempo 1D nanoLC system, then separated on a reversephase MagicTM C18 column (100 $\mu \mathrm{m}$ diameter, $15 \mathrm{~cm}$, MICHROM,USA), using a 120 min linear gradient of mobile phase A $\left(0.875 \% \mathrm{ACN} / 0.125 \% \mathrm{FA} / 99 \% \mathrm{H}_{2} \mathrm{O}\right)$ at a flow rate of $500 \mathrm{~nL} / \mathrm{min}$. The eluent was analyzed on a

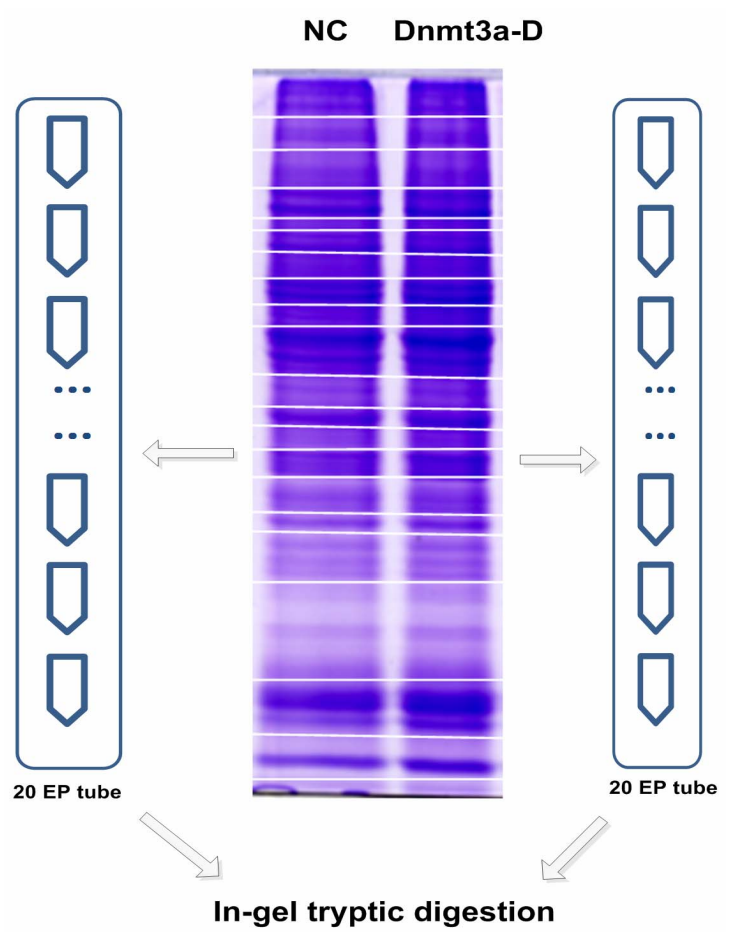

Figure 2. SDS-PAGE patterns of NC cell proteins and Dnmt3a - D cell proteins.
LTQ-orbitrap mass spectrometer (Thermo Electron, Bremen, Germany) equipped with a heated Desolvation Chamber Interface set to $200^{\circ} \mathrm{C}$ and operated using XCalibur software.

The LTQ-orbitrap operated in positive ion mode to survey full scan mass spectra (from m/z 385 to 2000). The most intense ten ions were selected for tandem mass spectrometry and the lock-mass option was used as described in previously published reports [21].

\subsection{Identification and Analysis of Proteins}

SEQUEST (V.28 (rev.12) 1998-2007) was used to identify proteins based on MS/MS spectra with Bioworks software (Rev.3.3.1 SP1, Thermo Scientific). We searched both forward and reverse sequences against the Swissport Mouse 090303 database in order to estimate the number of identifications that were false-positive in the sample.

The parameters for the SEQUEST search were comprised of the following criteria: Enzyme, trypsin; Missed cleavage sites, 2 ; fragment tolerance, $1.0 \mathrm{Da}$; peptide tolerance, 50.00 ppm; Numerical results, 250; Ion and Ion Series Calculated, b ion and y ion; Peptide matches, 10; Report duplicate peptide matches, 10. Oxidation of methionine, methylation of lysine, as well as phosphorylation of serine, threonine, and tyrosine were specified as variable modifications.

We inputted the DAT files from SEQUEST to the Trans-Proteomic Pipeline (TPP) v4.2 JETSTREAM rev 1 (ISB/SPC Proteomics Tools) and searched by allocating all DAT files in the biological sample. The parameters for peptide identification probability and protein identification probability were both set at 0.95 .

The results were exported and the proteins found to be altered in the Dnmt3a-D cell line as compared to the NC cell line were extracted by a program developed in-house. GO terms of altered proteins for cellular component and molecular function were searched and checked by Swiss-Prot (http://expasy.org/sprot/), NCBI (http://www. ncbi.nlm.nih.gov) and PIR (http://pir.georgetown.edu/ pirwww/index.shtml). The pathways of altered proteins were established using the KEGG mapper (http://www. genome.jp/kegg/.html). Demonstration of the role of altered proteins in metabolic channels and signal transduction pathways was considered significant.

\section{Results and Discussion}

\subsection{Cell Harvest}

Growth conditions of the two cell lines were optimized in our previously reported experiment [17] Cultured cells were harvested at a confluence level of 90\% (Fig- 
ure 3(a)).

Western blot analysis was employed to test the efficiency and specificity of the Dnmt3a-RNAi. The results demonstrated that stably transfected B16 Dnmt3a-D cells exhibited a remarkably low level of Dnmt3a expression. No change in Dnmt3a expression was observed in the control NC shRNA transfected cells. The expression of Dnmt3b was not affected in the NC or Dnmt3a-D cells, which is in concordance with our findings in our previous experiment (see Figure 3 (b) in [17]).

\subsection{Difference of Protein Expression between NC and Dnmt3a-D Cell Lines}

The NC and Dnmt3a-D cell line proteins identified were compared to determine their overlap (Figure 3(b)). There were 2413 proteins in common between the NC and Dnmt3a-D cell lines, and 467 were unique to the Dnmt3a-D cell line and were considered to be up-regulated proteins in the Dnmt3a-D cell line (Supplementary Materials, Table S1). There were 535 proteins unique to the NC cell line considered to be down-regulated proteins in the Dnmt3a-D cell line (Supplementary Materials, Table S2). The evidence suggests that Dnmt3a potentially either directly or indirectly regulates the expression of altered proteins.

\subsection{Genome Ontology of Altered Proteins}

In order to evaluate the effect of Dnmt3a depletion on protein expression and to explore the mechanism of Dnmt3a in tumorigenesis, the altered proteins were the principal targets for analysis. The genome ontology cellular localization of altered proteins was as follows: $35 \%$ of up-regulated proteins were localized to the cytoplasm, $24 \%$ to the nucleus, and $13 \%$ to the mitochondrion. The remainder of the up-regulated proteins were classified as golgi, plasma membrane, endoplasmic reticulum, cytoskeleton, extracellular, ribosome, others, and unclassified $(28 \%)$. The cellular localization of down-regulated proteins was found to be similar to the cellular localization of the up-regulated proteins (Figure 4(a), (b)). This result demonstrated that the altered proteins are primarily concentrated in the cytoplasm, nucleus, and mitochondria.

Among 467 up-regulated proteins, 161 exhibited an activity function (Figure 4(c)). Among the 535 downregulated proteins, 178 exhibited an activity function (Figure 4(d)).

There were a large number of altered proteins that exhibited catalytic activity and transferase activity, and they played important roles in the methylation, acetylation, glycosylation, and other epigenetic modifications of

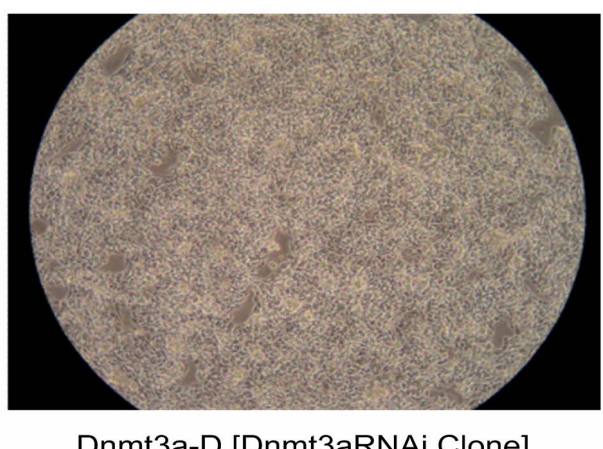

Dnmt3a-D [Dnmt3aRNAi Clone]

(a)

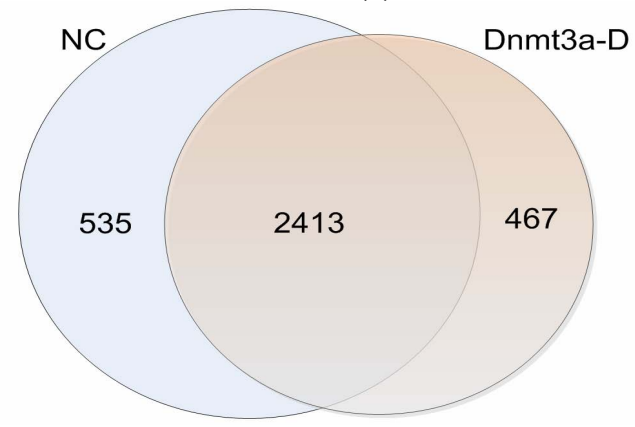

(b)

Figure 3. Cell Harvest and overlap of proteins between two cell lines. (a)The confluence of NC and Dnmt3a-D cell was more than $\mathbf{9 0 \%}$ after two days under microscope. (b)The proteins of $\mathrm{NC}$ and Dnmt3a-D cell line identified, and overlap of proteins between the two cell lines. 


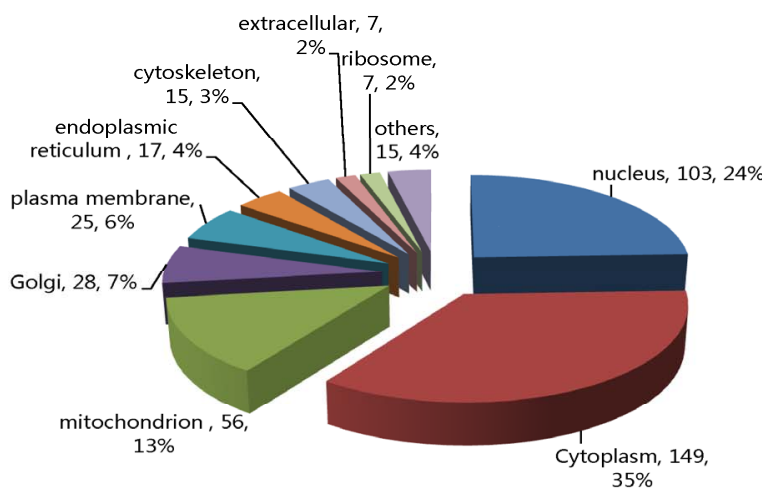

(a)

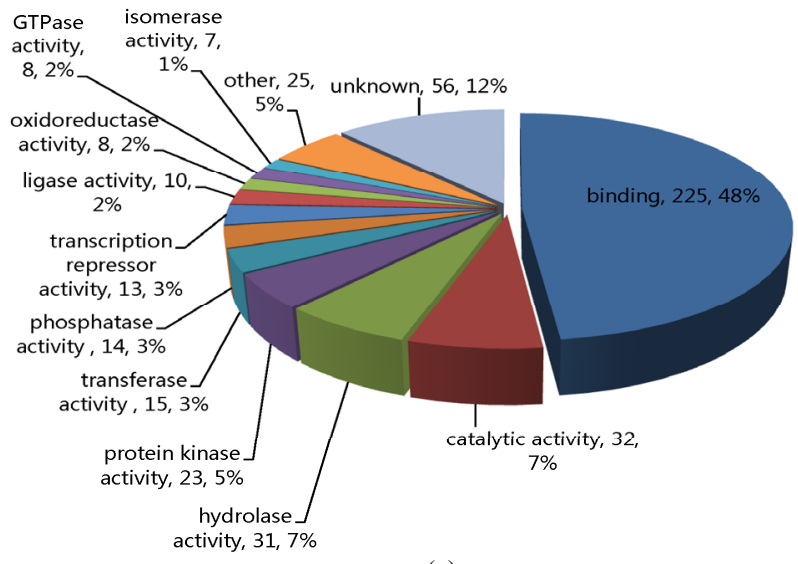

(c)

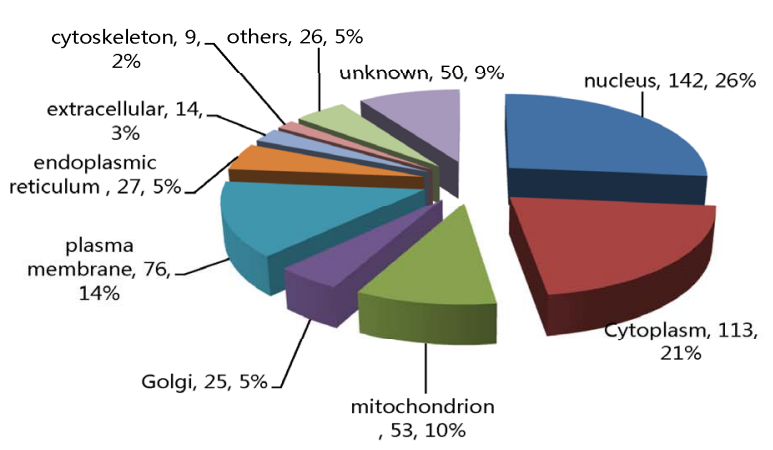

(b)

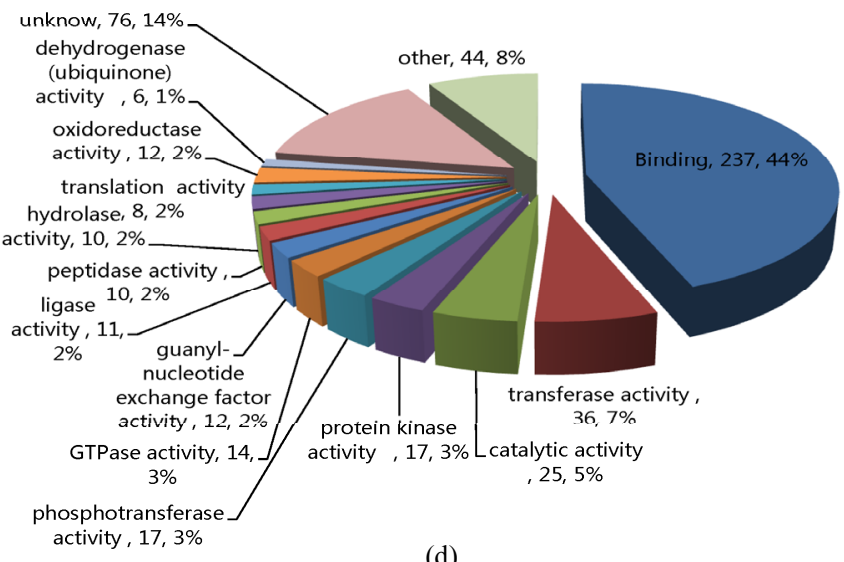

(d)

Figure. 4 Genome Ontology of altered Proteins. (A) Genome ontology cellular localization of up-regulated proteins. (B) Genome ontology cellular localization of down-regulated proteins. (C) Genome ontology molecular function of up-regulated proteins. (D) Genome ontology molecular function of down-regulated proteins.

the proteins. Another function that attracted our attention was phosphotransferase activity, which is essential for protein phosphorylation. It is well understood that protein phosphorylation is closely related to a variety of biological processes, such as DNA damage and repair [22], transcriptional regulation [23], signal transduction, and the regulation of apoptosis $[24,25]$.

\subsection{Pathway of Altered Proteins}

The altered proteins were grouped according to their respective KEGG pathway (Figure 5). The pathways were arranged according to the number of altered proteins. The pathways that included less than 10 altered proteins were not shown. The four pathways with the largest number of altered proteins were metabolic pathways (71 proteins), endocytosis (21 proteins), mitogen activated protein kinase signaling pathway (MAPK signaling pathway, 19 proteins) and pathways in cancer (17 proteins). Among these four pathways, the MAPK sig- naling pathway, closely related to the cancer and melanoma pathway, and which plays a key role in melanoma tumorigenesis, attracted our attention. The locations of altered proteins in the two pathway maps were established using the KEGG mapper.

\subsubsection{MAPK Signaling Pathway}

The altered proteins involved in the MAPK signaling pathway are listed in Table 1. The locations of the altered proteins involved in the MAPK signaling pathway are marked by pink boxes (up-regulated proteins) and yellow boxes (down-regulated proteins) as shown in Figure 6.

The MAPKs belong to a family of highly conserved kinases that convert extracellular signals to intracellular responses. MAPKs are unique to eukaryotes and are important signal transducing enzymes regulated by a phosphorylation cascade. Two upstream protein kinases are activated in the series that leads to the activation of a MAP kinase; additional kinases may also be required 
upstream of this three-kinase module. After activation, MAPKs phosphorylate specific serine and threonine residues of target substrates, including other protein kinases and many transcription factors. MAPKs are switched off by both generic phosphatases and dual- specificity phosphatases and are further regulated by scaffold proteins, which are usually specific for each of the three major mammalian MAPK pathways, including extracellular signal-regulated kinase (ERK), c-Jun Nterminal kinase (JNK), and p38 MAPK [18,26,27].

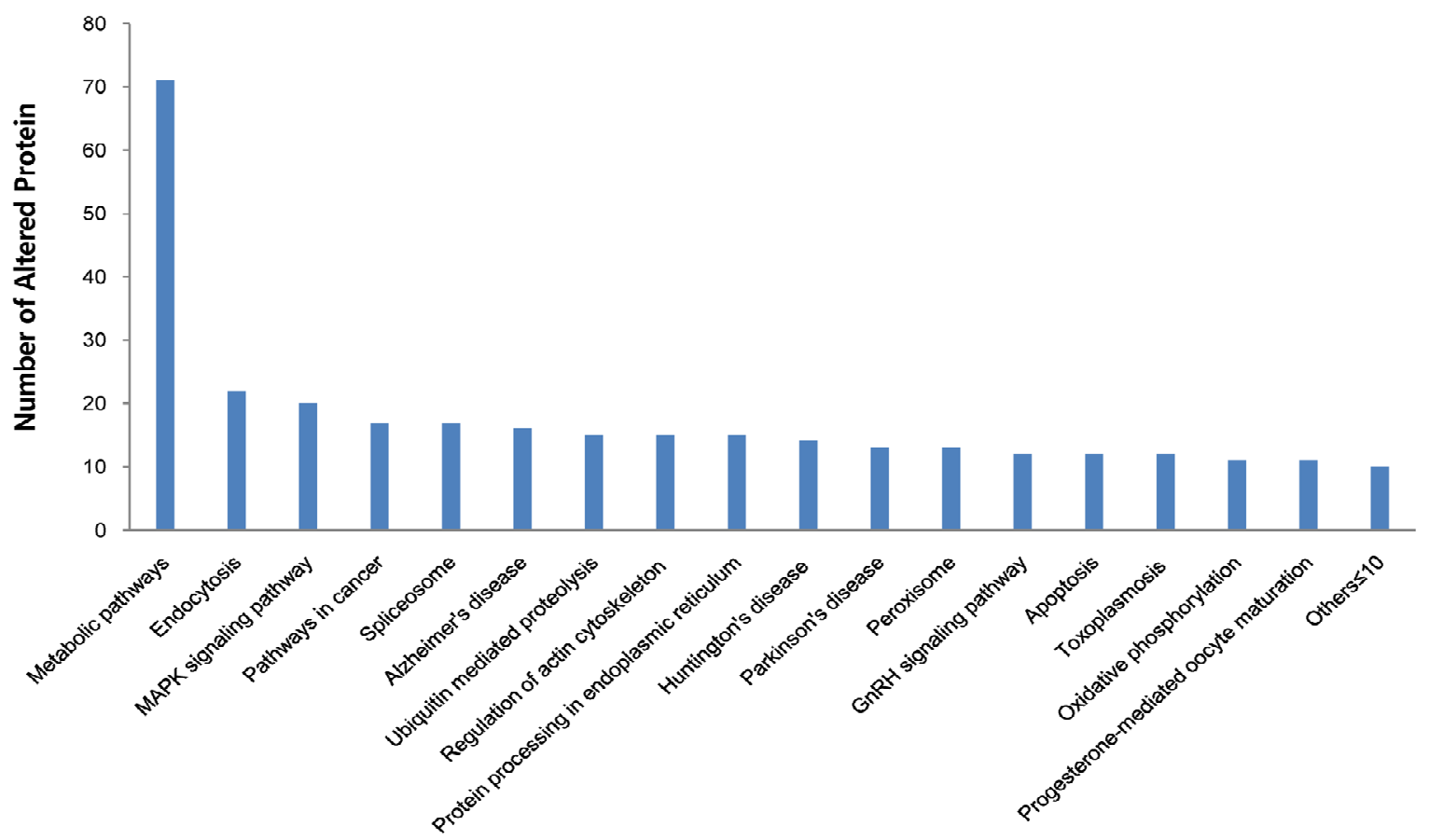

KEGG Pathway

Figure 5. The altered proteins were grouped by KEGG pathway.

Table 1. The altered proteins involved in MAPK signaling pathway.

\begin{tabular}{|c|c|c|c|c|}
\hline $\begin{array}{l}\text { Location in } \\
\text { Pathway }\end{array}$ & $\begin{array}{l}\text { Gen Bank } \\
\text { ID }\end{array}$ & $\begin{array}{l}\text { Gene } \\
\text { name }\end{array}$ & Synonyms & $\begin{array}{c}\text { Name of Altered Protein; } \\
\uparrow: \text { Up-regulated protein; } \downarrow: \text { Down-regulated protein }\end{array}$ \\
\hline \multirow{2}{*}{ PKA } & 18747 & Prkaca & Pkaca & cAMP-dependent protein kinase catalytic subunit alpha $\uparrow$ \\
\hline & 18749 & Prkacb & Pkacb & cAMP-dependent protein kinase catalytic subunit beta $\uparrow$ \\
\hline \multirow{2}{*}{ ERK } & 26413 & Mapk1 & Erk2, Mapk, Prkm1 & Mitogen-activated protein kinase $1 /$ ERK $2 \uparrow$ \\
\hline & 26417 & Mapk3 & Erk1, Prkm3 & Mitogen-activated protein kinase $3 / \mathrm{ERK} 1 \uparrow$ \\
\hline Ppp3c & 19058 & Ppp3r1 & $\mathrm{Cnb}$ & Calcineurin subunit B type $1 \uparrow$ \\
\hline CrkII & 12929 & Crkl & Crkol & Crk-like protein $\uparrow$ \\
\hline HGK & 26921 & Map4k4 & Nik & Mitogen-activated protein kinase kinase kinase kinase $4 \uparrow$ \\
\hline JNK & 26419 & Mapk8 & Jnk1, Prkm8 & Mitogen-activated protein kinase $8 / \mathrm{JNK} 1 \uparrow$ \\
\hline Cdc42Rac & 12540 & $\mathrm{Cdc} 42$ & 1 & Cell division control protein 42 homolog precursor $\uparrow$ \\
\hline MKK3 & 26397 & Map2k3 & Mkk3, Prkmk3 & Dual specificity mitogen-activated protein kinase kinase $3 \uparrow$ \\
\hline P38 & 26416 & Mapk14 & Crk1,Csbp1,Csbp2 & Mitogen-activated protein kinase $14 / \mathrm{p} 38 \alpha \uparrow$ \\
\hline PP2CB & 19043 & Ppm1b & Pp2c2, Pppm1b & Protein phosphatase $1 \mathrm{~B} \uparrow$ \\
\hline TAB1 & 66513 & Tab1 & Map3k7ip1 & TGF-beta-activated kinase 1 and MAP3K7-binding protein $1 \uparrow$ \\
\hline Rap1 & 109905 & Rapla & Krev-1 & Ras-related protein Rap-1A precursor $\downarrow$ \\
\hline RafB & 109880 & Braf & B-raf & Serine/threonine-protein kinase B-raf $\downarrow$ \\
\hline cPLA2 & 18783 & Pla2g4a & Cpla2, Pla2g4 & Cytosolic phospholipase A2 $\downarrow$ \\
\hline CASP & 12367 & Casp3 & Cpp32 & Caspase-3 precursor $\downarrow$ \\
\hline ECSIT & 26940 & Ecsit & Sitpec & mitochondrial precursor $\downarrow$ \\
\hline MKK6 & 26399 & Map2k6 & Prkmk6, Sapkk3 & Dual specificity mitogen-activated protein kinase kinase $6 \downarrow$ \\
\hline
\end{tabular}




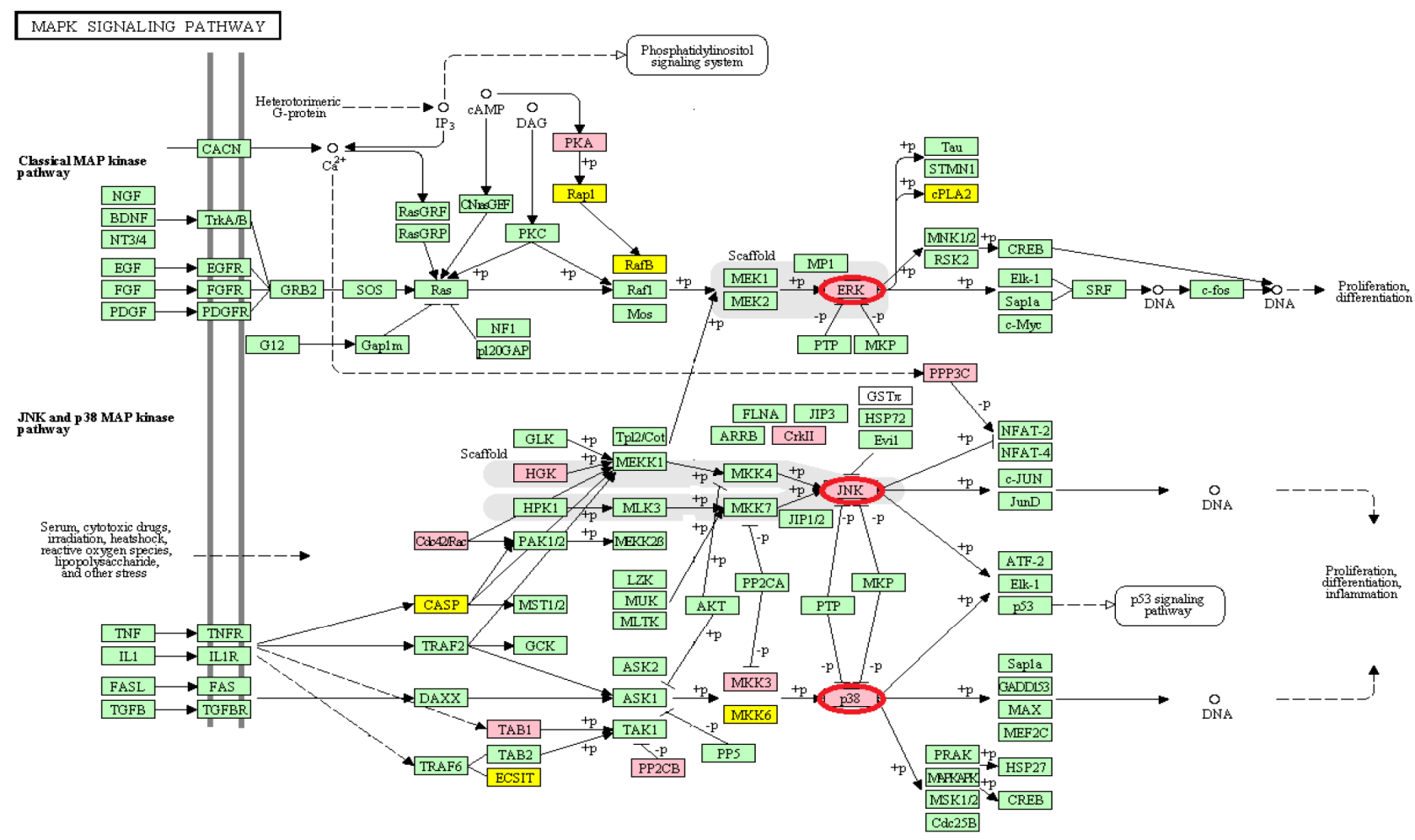

Figure 6. The location of altered proteins involved in MAPK signaling pathway from KEGG database.

In recent years, it has been demonstrated that the MAPK signaling pathway plays a key role in the cell cycle and gene expression regulation of various cells. MAP kinases are major components of the pathways that control embryogenesis, cell differentiation, cell proliferation, and cell death. Current research on the three pathways is described in detail, and offers insight into the mechanism of the pathways. Much of the review highlights research into the JNK and p38 MAPK pathways, stress activated protein kinase pathways that are also often deregulated in cancer. JNKs and p38 MAPKs are activated by environmental and genotoxic stress and are associated with tumorigenesis in humans and mice. The function of JNKs and p38 MAPKs in cancer development are complex and correlate with the wide range of cellular responses that they modulate [28,29].

\subsubsection{JNK Pathway}

Three genes encode the JNK proteins: MAPK8 encodes JNK1/ MAPK8, MAPK9 encodes JNK2/ MAPK9, and MAPK10 encodes JNK3/ MAPK10 [30]. Various JNKs differ substantially in their ability to interact with JUN, a well-established regulator of cell cycle progression [31]. A JNK2 deficiency results in elevated c-Jun phosphorylation and stability, whereas the absence of JNK1 reduces c-Jun phosphorylation and stability. JNK2 preferentially binds to c-Jun in unstimulated cells, thereby con- tributing to c-Jun degradation. In contrast, JNK1 becomes the major c-Jun interacting kinase after cell stimulation [32]. It has been recently demonstrated that the JNK pathway is linked to p53-dependent senescence via a conditional JNK1 allele [33]. The contribution of JNK1 to tumour development has also been investigated in mouse skin carcinogenesis. JNK1-knockout mice were shown to be more susceptible to tumours of the skin [34]. These results indicate that JNK1 may act as a suppressor of skin tumour development. In this study, JNK1 was found to be up-regulated in the Dnmt3a-D cell line which is unable to grow in-vivo, which potentially illustrates the fact that Dnmt3a depletion affects melanoma tumorigenesis by regulation of JNK1 expression.

\subsection{3. p38 MAPK Pathway}

The p38 MAPKs are activated by the upstream MKK3 and MKK6 kinases as shown in Figure 6. There are four genes that encode p38 MAPKs: MAPK14 encodes p38 $\alpha$ /MAPK14; MAPK11 encodes p38 $\beta /$ MAPK11; MAPK12 encodes p38 $\gamma$ /MAPK12; and MAPK13 encodes p38 /MAPK13 [35]. Among these, p38 $\alpha$ was found to be up-regulated in the Dnmt3a-D cell line. Most of the published studies that have investigated $\mathrm{p} 38$ MAPKs refer to p38 $\alpha$, as p38 $\alpha$ was highly abundant in most cell types [36]. A stress-activated protein kinase, $\mathrm{p} 38 \alpha$ suppresses tumor formation by negatively regulating cell cycle pro- 
gression and proliferation, or by inducing apoptosis [37-40].

There is evidence that indicates that $\mathrm{p} 38 \alpha$ also may be an important regulator of differentiation programs in many cell types, including epithelial lung cells and embryonic stem cells $[41,42]$. Moreover, p38 $\alpha$ directly phosphorylates and modulates the activity of several transcription factors involved in tissue-specific differentiation. The differentiation-inducing activity of $\mathrm{p} 38 \alpha$ may be related to tumour suppression, as p38 $\alpha$ activation triggers a high level of specific differentiation and lowers transformed phenotypes in renal carcinoma and colon cancer cell lines compared to cancer cell lines in which p38 $\alpha$ has not been activated [43-45]. The function of p38 $\alpha$ as a tumour suppressor in-vivo has been observed in a mouse model [18]. These results provide a reasonable explanation as to why the Dnmt3a-D cell line with p38a up-regulated is unable to grow in-vivo. Dnmt3a depletion potentially directly or indirectly regulates the expression of $\mathrm{p} 38 \alpha$.

\subsubsection{ERK Pathway}

ERK1 and ERK2 are ubiquitously expressed, although their relative abundance in tissues is variable. They are stimulated to a certain extent by a vast number of ligands and cellular perturbations, and there is evidence of some cell type specificity [46]. They are highly expressed in postmitotic neurons and other highly differentiated cells [47], and in these cells they are often involved in adaptive responses, such as long-term potentiation $[48,49]$. The receptor tyrosine kinase uses the ERK1 and ERK2 cell membrane signaling pathway [50-52]. Stimulation of these receptors by the appropriate ligand results is an increase in the catalytic activity of the receptor and subsequent autophosphorylation on tyrosine residues. Phosphorylation of these receptors results in the formation of multiprotein complexes whose organization dictates further downstream signaling events.

The ERK pathway is a major pathway involved in the control of growth signals, cell survival, and invasion.
ERK acts as a central point where multiple signaling pathways coalesce to drive transcription, and it plays a critical role in the pathway downstream of Ras, Raf, and MEK. Melanomas are known to harbour activation mutations for both Ras and BRAF, suggesting that the downstream effector ERK may be playing a key role in the oncogenic behaviour of these tumours. In-vitro studies have demonstrated that melanoma cell lines and tumour tissues exhibit high constitutive ERK activity. The high constitutive ERK activity in melanoma is most likely a consequence of mutations in the upstream components of the MAPK pathway $[53,54]$. In this study, we found that tumour cells cannot grow normally with ERK1 and ERK2 up-regulated while upstream components B-raf are concurrently down-regulated. Accordingly, we suspected that the activity of ERK1 and ERK2 was limited by the down-regulation of upstream components B-raf. This may account for the ability of Dnmt3a to affect the growth of melanoma by regulating the activity of ERK.

\subsubsection{Melanoma Pathway}

The altered proteins involved in the melanoma pathway are listed in Table 2. The locations of the altered proteins involved in the melanoma pathway are indicated with pink boxes (up-regulated proteins) and yellow boxes (down-regulated proteins) in Figure 7. The figure was manually edited. BRAF plays an important role in replicative and oncogene-induced senescence, as indicated by the red oval.

Frequent somatic mutation of BRAF is described in melanoma cell lines, such as the B16 mouse melanoma cell line, and other tumors [55,56]. BRAF is a serine/ threonine kinase that is commonly activated by a somatic point mutation in human cancer, and may provide new therapeutic opportunities for malignant melanoma [57]. Thus, we focused on BRAF in the melanoma pathway.

It has been reported that PTEN deficiency combined with BRAF activation induces a melanoma in-situ like phenotype without dermal invasion [58]. In our study,

Table 2. The altered proteins involved in melanoma pathway.

\begin{tabular}{ccccc}
\hline $\begin{array}{c}\text { Location in } \\
\text { Pathway }\end{array}$ & $\begin{array}{c}\text { Gen Bank } \\
\text { ID }\end{array}$ & Gene name & Synonyms & $\begin{array}{c}\text { Name of Altered Protein; } \\
\uparrow: \text { Up-regulated protein; } \downarrow: \text { Down-regulated protein }\end{array}$ \\
\hline ERK & 26413 & Mapk1 & Erk2, Mapk, Prkm1 & Mitogen-activated protein kinase 1 $\uparrow$ \\
BRAF & 26417 & Mapk3 & Erk1, Prkm3 & Mitogen-activated protein kinase 3 $\uparrow$ \\
Raf & 109880 & Braf & B-raf & Serine/threonine-protein kinase B-raf $\downarrow$ \\
CDK4/6 & 109880 & Braf & B-raf & Serine/threonine-protein kinase B-raf $\downarrow$ \\
& 12571 & Cdk6 & Crk2 & Cell division protein kinase $6 \downarrow$ \\
\hline
\end{tabular}


MELANOMA

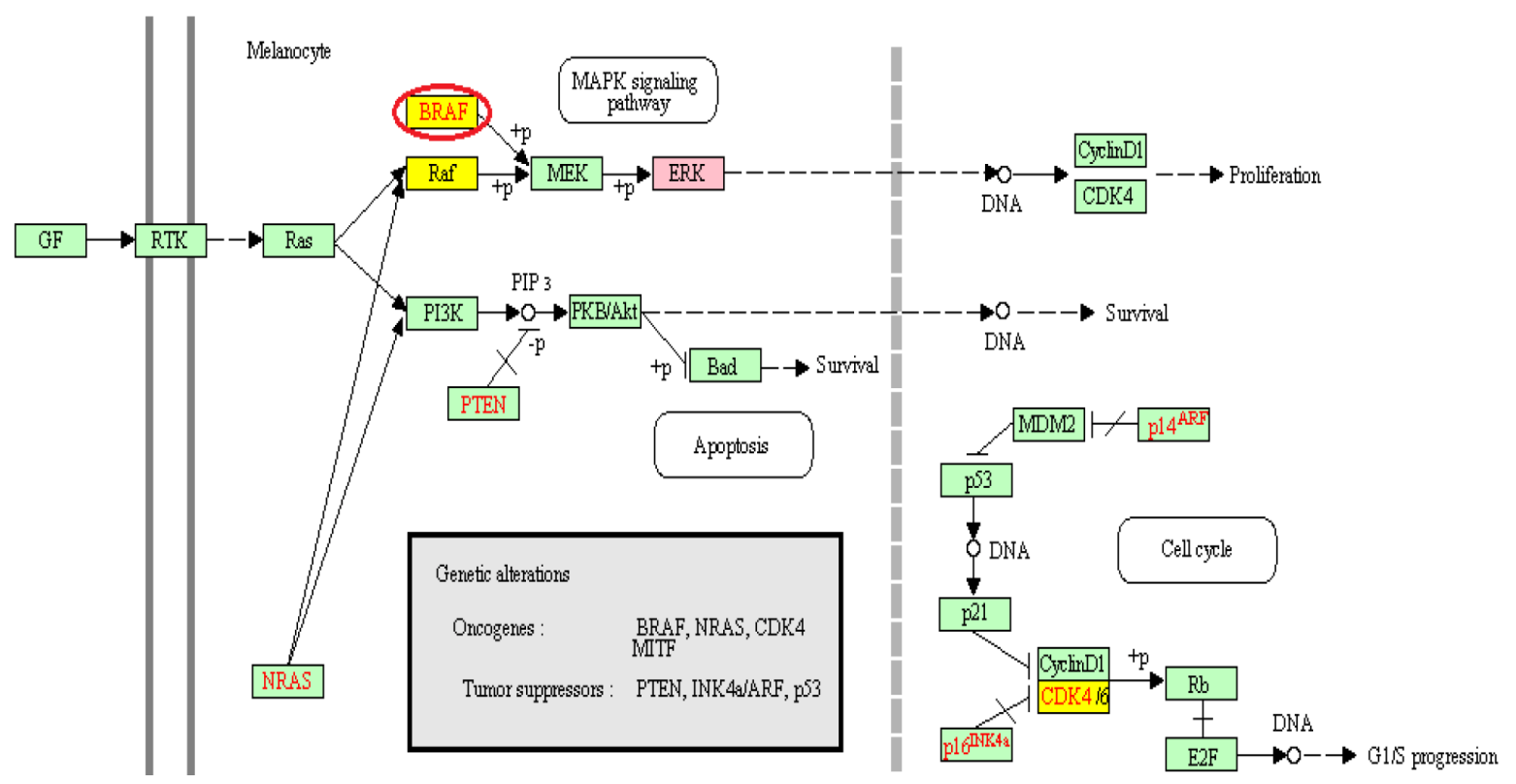

Figure 7. The location of altered proteins involved in melanoma pathway from KEGG database.

BRAF was found to be down-regulated in the melanoma pathway of the Dnmt3a-D cell line (Figure 7). Most likely, the down-regulated expression of BRAF resulted in the suppression of tumorigenesis. Dnmt3a depletion inhibited the growth of melanoma by directly or indirectly regulating the expression of BRAF.

\section{Conclusions}

This study demonstrates the varied changes in protein level, and that in the Dnmt3a-D cell line 467 proteins were up-regulated, while 535 proteins were down-regulated as compared to the $\mathrm{NC}$ cell line. GO analysis indicated that the altered proteins were primarily concentrated in the cytoplasm, nucleus, and mitochondrion, and that the altered proteins exhibiting activity function were primarily classified as exhibiting catalytic activity, transferase activity, and phosphotransferase activity. KEGG pathway analysis demonstrated that the MAPK signaling pathway exhibited a greater level of altered proteins, a fact that attracted our attention due to the close relationship with tumorigenesis. Taken together, our results strongly suggested that Dnmt3a depletion has a great impact on the expression of melanoma cell proteins. Additionally, Dnmt3a depletion may affect tumorigenesis through regulation of the proteins involved in tumorrelated pathways, such as the MAPK signaling pathway and the melanoma pathway. These results indicated that the tumor-related pathways may be potentially valuable for the treatment of malignant melanoma in the future.

\section{Acknowledgements}

The authors would like to acknowledge the financial support by the National Natural Science Foundation of China (20675057, 20705024).

\section{References}

[1] A. J. Miller and M. C. Jr Mihm, "Melanoma," The New England Journal of Medicine, Vol. 355, 2006, pp. 51-65.

[2] A. D. Sasse, E. C. Sasse, L. G. Clark, L. Ulloa and O. A. Clark, "Chemoimmunotherapy Versus Chemotherapy for Metastatic Malignant Melanoma," Cochrane Database System Reviews, 2007, p. D5413.

[3] M. F. Paz, M. F. Fraga, S. Avila, M. Guo, M. Pollan, J. G. Herman and M. Esteller, "A Systematic Profile of Dna Methylation in Human Cancer Cell Lines," Cancer Research, Vol. 63, 2003, pp. 1114-1121.

[4] W. M. Gallagher, O. E. Bergin, M. Rafferty, Z. D. Kelly, I. M. Nolan, E. J. Fox, A. C. Culhane, L. McArdle, M. F. Fraga, L. Hughes, C. A. Currid, F. O’Mahony, A. Byrne, A. A. Murphy, C. Moss, S. McDonnell, R. L. Stallings, J. A. Plumb, M. Esteller, R. Brown, P. A. Dervan and D. J. Easty, "Multiple Markers for Melanoma Progression Regulated by Dna Methylation: Insights From Transcriptomic Studies," Carcinogenesis, Vol. 26, No. 11, 2005, pp. 1856-1867.

[5] P. A. Van der Velden, W. Zuidervaart, M. H. Hurks, S. Pavey, B. R. Ksander, E. Krijgsman, R. R. Frants, C. P. Tensen, R. Willemze, M. J. Jager and N. A. Gruis, "Expression Profiling Reveals that Methylation of Timp3 is Involved in Uveal Melanoma Development," Interna- 
tional Journal of Cancer, Vol. 106, No. 4, 2003, pp. 472-479.

[6] E. Li, T. H. Bestor, and R. Jaenisch, "Targeted Mutation of the Dna Methyltransferase Gene Results in Embryonic Lethality," Cell, Vol. 69, No. 6, 1992, pp. 915-926.

[7] H. Lei, S. P. Oh, M. Okano, R. Juttermann, K. A. Goss, R. Jaenisch and E. Li, "De Novo Dna Cytosine Methyltransferase Activities in Mouse Embryonic Stem Cells," Development, Vol. 122, 1996, pp. 3195-3205.

[8] M. Okano, D. W. Bell, D. A. Haber and E. Li, "Dna Methyltransferases Dnmt3a and Dnmt3B are Essential for De Novo Methylation and Mammalian Development," Cell, Vol. 99, No, 3, 1999, pp. 247-257.

[9] K. D. Robertson, E. Uzvolgyi, G. Liang, C. Talmadge, J. Sumegi, F. A. Gonzales and P. A. Jones, "The Human Dna Methyltransferases (Dnmts) 1, 3a and 3B: Coordinate Mrna Expression in Normal Tissues and Overexpression in Tumors," Nucleic Acids Research, Vol. 27, No. 11, 1999, pp. 2291-2298.

doi:org/10.1093/nar/27.11.2291

[10] D. L. Zheng, L. Zhang, N. Cheng, X. Xu, Q. Deng, X. M. Teng, K. S. Wang, X. Zhang, J. Huang and Z. G. Han, "Epigenetic Modification Induced by Hepatitis B Virus X Protein Via Interaction with De Novo Dna Methyltransferase Dnmt3a," Journal of Hepatology, Vol. 50, No. 2, 2009, pp. 377-387. doi:org/10.1016/j.jhep.2008.10.019

[11] D. V. Maltseva, A. A. Baykov, A. Jeltsch, and E. S. Gromova, "Impact of 7,8-Dihydro-8-Oxoguanine On Methylation of the Cpg Site by Dnmt3a," Biochemistry, Vol. 48, No. 6, 2009, pp. 1361-1368.

[12] J. Feng, Y. Zhou, S. L. Campbell, Le T, E. Li, J. D. Sweatt, A. J. Silva and G. Fan, "Dnmt1 and Dnmt3a Maintain Dna Methylation and Regulate Synaptic Function in Adult Forebrain Neurons," Nature Neuroscience, Vol. 13, 2010, pp. 423-430. doi:org/10.1038/nn.2514

[13] H. Wu, V. Coskun, J. Tao, W. Xie, W. Ge, K. Yoshikawa, E. Li, Y. Zhang and Y. E. Sun, "Dnmt3a-Dependent Nonpromoter Dna Methylation Facilitates Transcription of Neurogenic Genes," Science, Vol. 329, No. 5990, 2010, pp. 444- 448.

[14] J. Nawarak, R. Huang-Liu, S. H. Kao, H. H. Liao, S. Sinchaikul, S. T. Chen and S. L. Cheng, "Proteomics Analysis of a375 Human Malignant Melanoma Cells in Response to Arbutin Treatment," Biochim Biophys Acta, Vol. 1794, No. 2, 2009, pp. 159-167.

[15] Y. Zhang, Y. Gao, G. Zhang, S. Huang, Z. Dong, C. Kong, D. Su, Du J, S. Zhu, Q. Liang, J. Zhang, J. Lu, and B. Huang, "Dnmt3a Plays a Role in Switches Between Doxorubicin-Induced Senescence and Apoptosis of Colorectal Cancer Cells," International Journal of Cancer, Vol. 128, No. 3, 2010, pp. 551-561.

[16] Z. Zhao, Q. Wu, J. Cheng, X. Qiu, J. Zhang and H. Fan, "Depletion of Dnmt3a Suppressed Cell Proliferation and Restored Pten in Hepatocellular Carcinoma Cell," Journal of Biomedicine and Biotechnology, Vol. 2010, Vol. 2010, pp. 737535-737545.

doi:org/10.1155/2010/737535
[17] T. Deng, Y. Kuang, L. Wang, J. Li, Z. Wang, and J. Fei, "An Essential Role for Dna Methyltransferase 3a in Melanoma Tumorigenesis," Biochemical and Biophysical Reseasch Communications, Vol. 387, No. 3, 2009, pp. 611-616. doi:org/10.1016/j.bbrc.2009.07.093

[18] E. F. Wagner and A. R. Nebreda, "Signal Integration by Jnk and P38 Mapk Pathways in Cancer Development," Nature Reviews Cancer, Vol. 9, 2009, pp. 537-549. doi:org/10.1038/nrc2694

[19] A. Zougman, B. Pilch, A. Podtelejnikov, M. Kiehntopf, C. Schnabel, C. Kumar and M. Mann, "Integrated Analysis of the Cerebrospinal Fluid Peptidome and Proteome," Journal of Proteome Research, Vol. 7, 2008, pp. 386-399. doi:org/10.1021/pr070501k

[20] B. Macek, L. F. Waanders, J. V. Olsen and M. Mann, "Top-Down Protein Sequencing and Ms3 On a Hybrid Linear Quadrupole Ion Trap-Orbitrap Mass Spectrometer," Mol Cell Proteomics, Vol. 5, 2006, pp. 949-958.

[21] J. V. Olsen, L. M. de Godoy, G. Li, B. Macek, P. Mortensen, R. Pesch, A. Makarov, O. Lange, S. Horning and M. Mann, "Parts Per Million Mass Accuracy On an Orbitrap Mass Spectrometer Via Lock Mass Injection Into a C-Trap," Mol Cell Proteomics, Vol. 4, 2005, pp. 2010-2021. doi:org/10.1074/mcp.T500030-MCP200

[22] S. K. Binz, A. M. Sheehan and M. S. Wold, "Replication Protein a Phosphorylation and the Cellular Response to Dna Damage," DNA Repair (Amst), Vol. 3, No. 8-9, 2004, pp. 1015-1024. doi:org/10.1016/j.dnarep.2004.03.028

[23] M. S. Kobor and J. Greenblatt, "Regulation of Transcription Elongation by Phosphorylation," Biochim Biophys Acta, Vol. 1577, No. 2, 2002, pp. 261-275.

[24] H. Ohkura, "Phosphorylation: Polo Kinase Joins an Elite Club," Current Biology, Vol. 13, 2003, pp. R912-R914. doi:org/10.1016/j.cub.2003.11.012

[25] P. P. Ruvolo, X. Deng and W. S. May, "Phosphorylation of Bcl2 and Regulation of Apoptosis," Leukemia, Vol. 15, No. 4, 2001, pp. 515-522.

[26] L. Chang and M. Karin, "Mammalian Map Kinase Signalling Cascades," Nature, Vol. 410, No. 6824, 2001, pp. $37-40$.

[27] J. M. Kyriakis and J. Avruch, "Mammalian MitogenActivated Protein Kinase Signal Transduction Pathways Activated by Stress and Inflammation," Physiological Reviews, Vol. 81, No. 2, 2001, pp. 807-869.

[28] A. R. Nebreda and A. Porras, "P38 Map Kinases: Beyond the Stress Response," Trends in Biochemical Sciences, Vol. 25, No. 6, 2000, pp. 257-260. doi:org/10.1016/S0968-0004(00)01595-4

[29] M. Karin and E. Gallagher, "From Jnk to Pay Dirt: Jun Kinases, their Biochemistry, Physiology and Clinical Importance," Iubmb Life, Vol. 57, No. 4-5, 2005, pp. 283-295.doi:org/10.1080/15216540500097111

[30] S. Gupta, T. Barrett, A. J. Whitmarsh, J. Cavanagh, H. K. Sluss, B. Derijard and R. J. Davis, "Selective Interaction of Jnk Protein Kinase Isoforms with Transcription Factors," EMBO Journal, Vol. 15, 1996, pp. 2760-2770.

[31] R. Eferl and E. F. Wagner, “Ap-1: A Double-Edged 
Sword in Tumorigenesis," Nature Reviews Cancer, Vol. 3, 2003, pp. 859-868. doi:org/10.1038/nrc1209

[32] K. Sabapathy, K. Hochedlinger, S. Y. Nam, A. Bauer, M. Karin and E. F. Wagner, "Distinct Roles for Jnk1 and Jnk2 in Regulating Jnk Activity and C-Jun-Dependent Cell Proliferation," Molecular Cell, Vol. 15, No. 5, 2004, pp. 713-725. doi:org/10.1016/j.molcel.2004.08.028

[33] M. Das, F. Jiang, H. K. Sluss, C. Zhang, K. M. Shokat, R. A. Flavell and R. J. Davis, "Suppression of P53-Dependent Senescence by the Jnk Signal Transduction Pathway," Proceedingof the Natlonal Academy Sciences of the US A, Vol. 104, 2007, pp. 15759-15764. doi:org/10.1073/pnas.0707782104

[34] D. Morse, L. E. Otterbein, S. Watkins, S. Alber, Z. Zhou, R. A. Flavell, R. J. Davis and A. M. Choi, "Deficiency in the C-Jun Nh2-Terminal Kinase Signaling Pathway Confers Susceptibility to Hyperoxic Lung Injury in Mice," American Journal of Physiology-Lung Cellular Molecular Physiology, Vol. 285, No. 1, 2003, pp. L250-L257.

[35] J. C. Lee, J. T. Laydon, P. C. McDonnell, T. F. Gallagher, S. Kumar, D. Green, D. McNulty, M. J. Blumenthal, J. R. Heys, S. W. Landvatter and A1. Et, "A Protein Kinase Involved in the Regulation of Inflammatory Cytokine Biosynthesis," Nature, Vol. 372, 1994, pp. 739-746.

[36] K. Ono and J. Han, "The P38 Signal Transduction Pathway: Activation and Function," Cellular Signalling, Vol. 12, No. 1, 2000, pp. 1-13. doi:org/10.1016/S0898-6568(99)00071-6

[37] D. V. Bulavin and AJ Jr Fornace, "P38 Map Kinase's Emerging Role as a Tumor Suppressor," Advances In Cancer Research, Vol. 92, 2004, pp. 95-118. doi:org/10.1016/S0065-230X(04)92005-2

[38] I. Dolado, A. Swat, N. Ajenjo, G. De Vita, A. Cuadrado and A. R. Nebreda, "P38Alpha Map Kinase as a Sensor of Reactive Oxygen Species in Tumorigenesis," Cancer Cell, Vol. 11, No. 2, 2007, pp. 191-205.

[39] F. B. Engel, M. Schebesta, M. T. Duong, G. Lu, S. Ren, J. B. Madwed, H. Jiang, Y. Wang and M. T. Keating, "P38 Map Kinase Inhibition Enables Proliferation of Adult Mammalian Cardiomyocytes," Genes Development, Vol. 19, 2005, pp. 1175-1187. doi:org/10.1101/gad.1306705

[40] L. J. Hui, L. Bakiri, A. Mairhorfer, N. Schweifer, C. Haslinger, L. Kenner, V. Komnenovic, H. Scheuch, H. Beug and E. F. Wagner, "P38X3B1; Suppresses Normal and Cancer Cell Proliferation by Antagonizing the JnkX2013;C-Jun Pathway," Nature Genetics, Vol. 39, 2007.

[41] A. Jones and A. Charlton, "Determination of Metaldehyde in Suspected Cases of Animal Poisoning Using Gas Chromatography-Ion Trap Mass Spectrometry," Journal Agric Food Chemistry, Vol. 47, No. 11, 1999, pp. 4675-4677. doi:org/10.1021/jf990026d

[42] X. Wang, C. H. Goh and B. Li, "P38 Mitogen-Activated Protein Kinase Regulates Osteoblast Differentiation through Osterix," Endocrinology, Vol. 148, 2007, pp. 1629-1637.

[43] C. Hikita, S. Vijayakumar, J. Takito, H. ErdjumentBromage, P. Tempst and Q. Al-Awqati, "Induction of
Terminal Differentiation in Epithelial Cells Requires Polymerization of Hensin by Galectin 3," Journal of Cell Biology, Vol. 151, No. 6, 2000, pp. 1235-1246. doi:org/10.1083/jcb.151.6.1235

[44] P. Ordonez-Moran, M. J. Larriba, H. G. Palmer, R. A. Valero, A. Barbachano, M. Dunach, A. G. de Herreros, C. Villalobos, M. T. Berciano, M. Lafarga and A. Munoz, "Rhoa-Rock and P38Mapk-Msk1 Mediate Vitamin D Effects On Gene Expression, Phenotype and Wnt Pathway in Colon Cancer Cells," Journal of Cell Biology, Vol. 183, No. 4, 2008, pp. 697-710. doi:org/10.1083/jcb.200803020

[45] G. J. Finn, B. S. Creaven and D. A. Egan, "Daphnetin Induced Differentiation of Human Renal Carcinoma Cells and its Mediation by P38 Mitogen-Activated Protein Kinase," Biochemical Pharmacology, Vol. 67, 2004, pp. 1779-1788. doi:org/10.1016/j.bcp.2004.01.014

[46] T. S. Lewis, P. S. Shapiro and N. G. Ahn, "Signal Transduction through Map Kinase Cascades," Advances In Cancer Research, Vol. 74, 1998, pp. 49-139. doi:org/10.1016/S0065-230X(08)60765-4

[47] T. G. Boulton, S. H. Nye, D. J. Robbins, N. Y. Ip, E. Radziejewska, S. D. Morgenbesser, R. A. DePinho, N. Panayotatos, M. H. Cobb and G. D. Yancopoulos, "Erks: A Family of Protein-Serine/Threonine Kinases that are Activated and Tyrosine Phosphorylated in Response to Insulin and Ngf," Cell, Vol. 65, 1991, pp. 663-675.

[48] C. M. Atkins, J. C. Selcher, J. J. Petraitis, J. M. Trzaskosand J. D. Sweatt, "The Mapk Cascade is Required for Mammalian Associative Learning," Nature Neuroscience, Vol. 1, 1998, pp. 602-609. doi:org/10.1038/2836

[49] R. Brambilla, N. Gnesutta, L. Minichiello, G. White, A. J. Roylance, C. E. Herron, M. Ramsey, D. P. Wolfer, V. Cestari, C. Rossi-Arnaud, S. G. Grant, P. F. Chapman, H. P. Lipp, E. Sturani and R. Klein, "A Role for the Ras Signalling Pathway in Synaptic Transmission and LongTerm Memory," Nature, Vol. 390, 1997, PP. 281-286.

[50] G. Pearson, F. Robinson, Gibson T. Beers, B. E. Xu, M. Karandikar, K. Berman and M. H. Cobb, "Mitogen-Activated Protein (Map) Kinase Pathways: Regulation and Physiological Functions," Endocrine Reviews, Vol. 22, No. 2, 2001, pp. 153-183. doi:org/10.1210/er.22.2.153

[51] T. Pawson and J. D. Scott, "Signaling through Scaffold, Anchoring, and Adaptor Proteins," Science, Vol. 278, No. 5346, 1997, pp. 2075-2080.

[52] T. Hunter, "Protein Kinases and Phosphatases: The Yin and Yang of Protein Phosphorylation and Signaling," Cell, Vol. 80, No. 2, 1995, pp. 225-236.

[53] A. M. Aronov, C. Baker, G. W. Bemis, J. Cao, G. Chen, P. J. Ford, U. A. Germann, J. Green, M. R. Hale, M. Jacobs, J. W. Janetka, F. Maltais, G. Martinez-Botella, M. N. Namchuk, J. Straub, Q. Tang and X. Xie, "Flipped Out: Structure-Guided Design of Selective Pyrazolylpyrrole Erk Inhibitors," Journal of Medicinal Chemistry, Vol. 50, No. 5, 2007, pp. 1280-1287. doi:org/10.1021/jm061381f

[54] K. S. Smalley, "A Pivotal Role for Erk in the Oncogenic Behaviour of Malignant Melanoma?" International 
Journal of Cancer, Vol. 104, No. 5, 2003, pp. 527-532. doi:org/10.1002/ijc.10978

[55] H. Gear, H. Williams, E. G. Kemp and F. Roberts, "Braf Mutations in Conjunctival Melanoma," Invest Ophthalmology Visual Science, Vol. 45, No. 8, 2004, pp. 24842488. doi:org/10.1167/iovs.04-0093

[56] V. K. Goel, A. J. Lazar, C. L. Warneke, M. S. Redston and F. G. Haluska, "Examination of Mutations in Braf, Nras, and Pten in Primary Cutaneous Melanoma," Journal of Investigative Dermatology, Vol. 126, 2006, pp. 154-160.

[57] H. Davies, G. R. Bignell, C. Cox, P. Stephens, S. Edkins, S. Clegg, J. Teague, H. Woffendin, M. J. Garnett, W. Bottomley, N. Davis, E. Dicks, R. Ewing, Y. Floyd, K. Gray, S. Hall, R. Hawes, J. Hughes, V. Kosmidou, A.
Menzies, C. Mould, A. Parker, C. Stevens, S. Watt, S. Hooper, R. Wilson, H. Jayatilake, B. A. Gusterson, C. Cooper, J. Shipley, D. Hargrave, K. Pritchard-Jones, N. Maitland, G. Chenevix-Trench, G. J. Riggins, D. D. Bigner, G. Palmieri, A. Cossu, A. Flanagan, A. Nicholson, J. W. Ho, S. Y. Leung, S. T. Yuen, B. L. Weber, H. F. Seigler, T. L. Darrow, H. Paterson, R. Marais, C. J. Marshall, R. Wooster, M. R. Stratton and P. A. Futreal, "Mutations of the Braf Gene in Human Cancer," Nature, Vol. 417, No. 6892, 2002, pp. 949-954.

[58] R. S. Lo and O. N. Witte, "Transforming Growth Factor-Beta Activation Promotes Genetic Context-Dependent Invasion of Immortalized Melanocytes," Cancer Research, Vol. 68, No. 4248, 2008, pp. 4248-4257. doi:org/10.1158/0008-5472.CAN-07-5671

\section{Supporting Information Available}

There are 467 proteins were up-regulated (Supplementary Materials, Table S1) and 535 proteins were down-regulated (Supplementary Materials, Table S2) in the Dnmt3a-D cell line as compared with the NC cell line. Supplementary materials Table S3 including the abbreviated words and their full name.

Table S1

\begin{tabular}{|c|c|c|c|c|}
\hline protein & $\begin{array}{l}\text { Protein } \\
\text { probability }\end{array}$ & $\begin{array}{l}\text { num unique } \\
\text { peps }\end{array}$ & $\begin{array}{l}\text { precursor ion } \\
\text { charge }\end{array}$ & peptide sequence \\
\hline sp|Q8CGP0|H2B3B_MOUSE & 0.9997 & 2 & 2 & AMGIMNSFVNDIFER \\
\hline sp|Q8QZT1|THIL_MOUSE & 1 & 18 & 2 & ASKPTLNEVVIVSAIR \\
\hline sp|Q9QZQ8|H2AY_MOUSE & 1 & 9 & 2 & AASADSTTEGTPTDGFTVLSTK \\
\hline sp|O88544|CSN4_MOUSE & 1 & 11 & 2 & AIQLSGTEQLEALK \\
\hline sp|Q9CR16|PPID_MOUSE & 1 & 15 & 2 & DGSGDSHPDFPEDADIDLK \\
\hline sp|Q9WVJ2|PSD13_MOUSE & 1 & 10 & 2 & LYENFISEFEHR \\
\hline sp|P40336|VP26A_MOUSE & 1 & 11 & 2 & ELALPGELTQSR \\
\hline sp|Q921H8|THIKA_MOUSE & 1 & 12 & 2 & AEELGLPILGVLR \\
\hline sp|P63085|MK01_MOUSE & 1 & 12 & 2 & FDM[147]ELDDLPK \\
\hline sp|Q9QWL7|K1C17_MOUSE & 1 & 5 & 2 & LLEGEDAHLTQYK \\
\hline sp|Q99J62|RFC4_MOUSE & 1 & 10 & 2 & AITFLQSATR \\
\hline sp|Q60737|CSK21_MOUSE & 1 & 11 & 2 & GGPNIITLADIVK \\
\hline sp|Q91WK2|EIF3H_MOUSE & 1 & 11 & 2 & LFM[147]AQALQEYNN \\
\hline sp|O54984|ARSA1_MOUSE & 1 & 8 & 2 & GM[147]NFSVVVFDTAPTGH \\
\hline sp|Q07417|ACADS_MOUSE & 1 & 10 & 2 & GISAFLVPM[147]PTPGLTLGK \\
\hline sp|Q99KV1|DJB11_MOUSE & 1 & 7 & 2 & FQDLGAAYEVLSDSEK \\
\hline sp|Q9DAR7|DCPS_MOUSE & 1 & 9 & 2 & IVFENPDPSDGFVLIPDLK \\
\hline sp|Q9R1T2|SAE1_MOUSE & 1 & 9 & 2 & AQNLNPM[147]VDVK \\
\hline sp|Q9D0M1|KPRA_MOUSE & 1 & 10 & 2 & GQDIFIIQTIPR \\
\hline sp|Q9JHJ0|TMOD3_MOUSE & 1 & 7 & 2 & DLGDYKDLDEDELLGK \\
\hline sp|Q9Z2K1|K1C16_MOUSE & 1 & 4 & 2 & TRLEQEIATYR \\
\hline sp|Q9Z1G3|VATC1_MOUSE & 1 & 11 & 2 & VAQYM[147]ADVLEDSKDK \\
\hline
\end{tabular}




\begin{tabular}{|c|c|c|c|c|}
\hline sp|P70362|UFD1_MOUSE & 1 & 7 & 2 & FQPQSPDFLDITNPK \\
\hline sp|P47811|MK14_MOUSE & 1 & 9 & 2 & HENVIGLLDVFTPAR \\
\hline sp|Q9R0U0|FUSIP_MOUSE & 1 & 6 & 2 & GFAYVQFEDVR \\
\hline sp|P63276|RS17_MOUSE & 1 & 3 & 2 & IAGYVTHLM[147]K \\
\hline sp|Q9CXW2|RT22_MOUSE & 1 & 8 & 2 & LM[147]TQAQLEEATR \\
\hline sp|Q6Y7W8|PERQ2_MOUSE & 1 & 9 & 2 & EVESPYEVHDYTR \\
\hline sp|Q9CPX6|ATG3_MOUSE & 1 & 5 & 2 & LWLFGYDEQR \\
\hline sp|P70318|TIAR_MOUSE & 1 & 4 & 2 & FEDVVNQSSPK \\
\hline sp|P05132|KAPCA_MOUSE & 1 & 9 & 2 & ILQAVNFPFLVK \\
\hline sp|Q9DBS1|TMM43_MOUSE & 1 & 9 & 2 & LLSDPNYGVHLPAVK \\
\hline sp|P54923|ADPRH_MOUSE & 1 & 7 & 3 & DGETIHQQLAQM[147]GDLEAIDVAR \\
\hline sp|Q9D7G0|PRPS1_MOUSE & 1 & 6 & 2 & IFSGSSHQDLSQK \\
\hline sp|Q9ER00|STX12_MOUSE & 1 & 4 & 2 & ELGSLPLPLSASEQR \\
\hline sp|Q9ER88|RT29_MOUSE & 1 & 6 & 2 & FDQPLEASTWLK \\
\hline sp|Q9Z0G0|GIPC1_MOUSE & 1 & 6 & 2 & APPLVENEEAEPSR \\
\hline sp|Q99LG2|TNPO2_MOUSE & 1 & 4 & 2 & ALVM[147]LLEVR \\
\hline sp|Q9CQT1|EI2BL_MOUSE & 1 & 6 & 2 & AGAGGPGLAALVAFVR \\
\hline sp|Q9WUL7|ARL3_MOUSE & 1 & 6 & 2 & LNVWDIGGQR \\
\hline sp|P47964|RL36_MOUSE & 0.9975 & 2 & 2 & YPM[147]AVGLNK \\
\hline sp|P26516|PSD7_MOUSE & 1 & 5 & 2 & TNDQM[147]VVVYLASLIR \\
\hline sp|Q8K157|GALM_MOUSE & 1 & 4 & 2 & VSPDGEEGYPGELK \\
\hline sp|P58774|TPM2_MOUSE & 0.9994 & 1 & 2 & ATDAEADVASLNR \\
\hline sp|P61963|WDR68_MOUSE & 1 & 3 & 2 & DM[147]FASVGADGSVR \\
\hline sp|Q64442|DHSO_MOUSE & 1 & 4 & 2 & AM[147]GAAQVVVTDLSASR \\
\hline sp|Q99J09|MEP50_MOUSE & 1 & 4 & 2 & IWDLAQQVSLNSYR \\
\hline sp|Q9CX34|SUGT1_MOUSE & 1 & 6 & 2 & DYASALETFAEGQK \\
\hline sp|Q9QYA2|TOM40_MOUSE & 1 & 6 & 2 & FVNWQVDGEYR \\
\hline sp|Q9QYJ3|DNJB1_MOUSE & 1 & 6 & 2 & DGSDVIYPAR \\
\hline sp|P68181|KAPCB_MOUSE & 0.9987 & 1 & 2 & ILQAVEFPFLVR \\
\hline sp|Q60766|IRGM_MOUSE & 1 & 5 & 2 & DLSTSVLSEVR \\
\hline sp|Q64213|SF01_MOUSE & 1 & 4 & 3 & ILRPWQSSETR \\
\hline sp|Q8R323|RFC3_MOUSE & 1 & 7 & 2 & AIYHLEAFVAK \\
\hline sp|Q8R574|KPRB_MOUSE & 1 & 5 & 2 & IFVM[147]ATHGLLSSDAPR \\
\hline sp|Q99JB2|STML2_MOUSE & 1 & 4 & 2 & AEQINQAAGEASAVLAK \\
\hline sp|Q9EQ80|NIF3L_MOUSE & 1 & 5 & 2 & TLM[147]QVLAFLSQDR \\
\hline sp|Q9JLC8|SACS_MOUSE & 1 & 9 & 2 & LLLVLNK \\
\hline sp|O09174|AMACR_MOUSE & 1 & 5 & 2 & GQNILDGGAPFYTTYK \\
\hline sp|O35435|PYRD_MOUSE & 1 & 7 & 2 & QTQLTTDGLPLGINLGK \\
\hline sp|P24288|BCAT1_MOUSE & 1 & 5 & 2 & HLTM[147]DDLATALEGNR \\
\hline sp|P70697|DCUP_MOUSE & 1 & 5 & 2 & LVQQM[147]LDDFGPQR \\
\hline sp|Q61187|TS101_MOUSE & 1 & 5 & 2 & ELVNLTGTIPVR \\
\hline sp|Q6PD19|CJ076_MOUSE & 1 & 6 & 2 & LQDGLDQYER \\
\hline sp|Q91WM2|CECR5_MOUSE & 1 & 5 & 2 & QM[147]LVSGQGPLVENAR \\
\hline sp|P18872|GNAO_MOUSE & 0.9998 & 2 & 2 & TTGIVETHFTFK \\
\hline sp|P47941|CRKL_MOUSE & 1 & 4 & 2 & IHYLDTTTLIEPAPR \\
\hline sp|Q8VDT9|RM50_MOUSE & 1 & 5 & 2 & DVLDFYNVPVQDK \\
\hline sp|Q99M71|EPDR1_MOUSE & 1 & 3 & 2 & ALVSYDGLNQR \\
\hline
\end{tabular}




\begin{tabular}{|c|c|c|c|c|}
\hline sp|Q9WTQ8|TIM23_MOUSE & 1 & 3 & 2 & NVQILNM[147]VTR \\
\hline sp|Q80WQ2|VAC14_MOUSE & 1 & 4 & 2 & DFVAQNNTM[147]QIK \\
\hline sp|Q9CQ92|FIS1_MOUSE & 1 & 2 & 2 & GLLQTEPQNNQAK \\
\hline sp|Q9CXE7|TMED5_MOUSE & 1 & 4 & 2 & LEDILESINSIK \\
\hline sp|Q9DC50|OCTC_MOUSE & 1 & 5 & 2 & AASDLQIAASTFTSFGK \\
\hline sp|Q9JK48|SHLB1_MOUSE & 1 & 4 & 2 & LAADAGTFLSR \\
\hline sp|Q8K0D0|PCTK2_MOUSE & 1 & 4 & 2 & LDSEGIELITK \\
\hline sp|Q99J95|CDK9_MOUSE & 0.9997 & 3 & 2 & LADFGLAR \\
\hline sp|P21278|GNA11_MOUSE & 1 & 6 & 2 & IATVGYLPTQQDVLR \\
\hline sp|Q8BMS9|RASF2_MOUSE & 1 & 5 & 2 & TSVFTPAYGSVTNVR \\
\hline sp|Q99JT2|MST4_MOUSE & 1 & 3 & 2 & LADFGVAGQLTDTQIK \\
\hline sp|O70493|SNX12_MOUSE & 0.9966 & 2 & 2 & TNLPIFK \\
\hline sp|O08915|AIP_MOUSE & 1 & 3 & 2 & VLELDPALAPVVSR \\
\hline sp|P59708|PM14_MOUSE & 1 & 3 & 2 & GTAYVVYEDIFDAK \\
\hline sp|P70404|IDH3G_MOUSE & 1 & 6 & 2 & ENTEGEYSSLEHESVAGVVESLK \\
\hline sp|Q80V26|IMPA3_MOUSE & 1 & 3 & 2 & EM[147]LAVAVLAAER \\
\hline sp|Q8BGR9|UBCP1_MOUSE & 1 & 4 & 2 & LDDFLELNHK \\
\hline sp|Q8BTZ7|GMPPB_MOUSE & 1 & 5 & 2 & ALILVGGYGTR \\
\hline sp|Q8N9S3|AHSA2_MOUSE & 1 & 4 & 2 & LQASPVALGVR \\
\hline sp|Q91YM2|GRLF1_MOUSE & 1 & 6 & 2 & EQLTEGEEIAQEIDGR \\
\hline sp|Q99JF8|PSIP1_MOUSE & 1 & 4 & 2 & QVDTEEAGM[147]VTAATASNVK \\
\hline sp|Q9CQR4|THEM2_MOUSE & 1 & 2 & 2 & TLAFASVDLTNK \\
\hline sp|Q9CXY9|GPI8_MOUSE & 1 & 3 & 2 & NVLITDFFGSVR \\
\hline sp|Q9JHI5|IVD_MOUSE & 1 & 4 & 2 & IGQFQLM[147]QGK \\
\hline sp|Q9WVL0|MAAI_MOUSE & 1 & 2 & 2 & VITSGFNALEK \\
\hline sp|O08583|THOC4_MOUSE & 1 & 3 & 2 & QQLSAEELDAQLDAYNAR \\
\hline sp|Q63844|MK03_MOUSE & 1 & 3 & 2 & FDM[147]ELDDLPK \\
\hline sp|Q00899|TYY1_MOUSE & 1 & 3 & 2 & TLEGEFSVTM[147]WSSDEKK \\
\hline sp|Q3URS9|CCD51_MOUSE & 1 & 4 & 2 & EDNQYLELATLEHR \\
\hline sp|Q8R2U6|NUDT4_MOUSE & 1 & 2 & 2 & LLGIFENQDR \\
\hline sp|P13011|ACOD2_MOUSE & 0.9913 & 1 & 2 & DDLYDPTYQDDEGPPPK \\
\hline sp|O70439|STX7_MOUSE & 1 & 2 & 2 & TLNQLGTPQDSPELR \\
\hline sp|O88543|CSN3_MOUSE & 1 & 2 & 2 & AM[147]DQEITVNPQFVQK \\
\hline sp|P47199|QOR_MOUSE & 1 & 3 & 2 & VFEFGGPEVLK \\
\hline sp|Q8BG94|COMD7_MOUSE & 1 & 3 & 2 & FGVTSGSSELEK \\
\hline sp|Q8BH69|SPS1_MOUSE & 1 & 4 & 2 & IIEVAPQVATQNVNPTPGATS \\
\hline sp|Q8CAY6|THIC_MOUSE & 1 & 5 & 2 & ILVTLLHTLER \\
\hline sp|Q8K2Z2|PRP39_MOUSE & 1 & 4 & 2 & DLLTGEQFIQLR \\
\hline sp|Q8R349|CDC16_MOUSE & 1 & 4 & 2 & YAEALDYHR \\
\hline sp|Q8VCG3|WDR74_MOUSE & 1 & 3 & 2 & VWDLQGSEEPVFR \\
\hline sp|Q921X9|PDIA5_MOUSE & 1 & 4 & 2 & FHISAFPTLK \\
\hline sp|Q99K23|UFSP2_MOUSE & 1 & 4 & 2 & INAYHFPDELYK \\
\hline sp|Q99LD9|EI2BB_MOUSE & 1 & 4 & 2 & FVAPEEVLPFTEGDILEK \\
\hline sp|Q9D2R6|CCD56_MOUSE & 1 & 3 & 2 & FLDELEDEAK \\
\hline sp|Q9D8X5|CNOT8_MOUSE & 1 & 3 & 2 & GGLQEVADQLDLQR \\
\hline sp|Q9DCA5|BXDC2_MOUSE & 1 & 4 & 2 & EFLIQIFSTPR \\
\hline sp|P21279|GNAQ_MOUSE & 1 & 2 & 2 & M[147]FVDLNPDSDK \\
\hline
\end{tabular}




\begin{tabular}{|c|c|c|c|c|}
\hline sp|Q8VE80|THOC3_MOUSE & 0.9992 & 2 & 2 & YVLGM[147]QELFR \\
\hline sp|Q62422|OSTF1_MOUSE & 0.9989 & 2 & 2 & ALYTFEPR \\
\hline sp|P61294|RAB6B_MOUSE & 0.9979 & 1 & 2 & GSDVIIMLVGNK \\
\hline sp|Q8R2E9|ERO1B_MOUSE & 0.9969 & 1 & 2 & TLLLSIFQDTK \\
\hline sp|O09110|MP2K3_MOUSE & 1 & 2 & 2 & FPYESWGTPFQQLK \\
\hline sp|O35658|C1QBP_MOUSE & 1 & 2 & 2 & AFVEFLTDEIKEEK \\
\hline sp|P62488|RPB7_MOUSE & 1 & 3 & 2 & GEVVDAVVTQVNK \\
\hline sp|Q59J78|MIMIT_MOUSE & 1 & 3 & 2 & ETSEELLPSPTATQVK \\
\hline sp|Q60866|PTER_MOUSE & 1 & 3 & 2 & GGGALVENTTTGLSR \\
\hline sp|Q61249|IGBP1_MOUSE & 1 & 3 & 2 & AAGM[147]LSQLDLFSR \\
\hline sp|Q62203|SF3A2_MOUSE & 1 & 4 & 2 & M[147]EKPPAPPSLPAGPPGVK \\
\hline sp|Q78HU3|F125A_MOUSE & 1 & 2 & 2 & GPLPSGFSAVNDPQDIK \\
\hline sp|Q78JW9|UBFD1_MOUSE & 1 & 4 & 2 & IM[147]VVGSTINDVLAVNTPK \\
\hline sp|Q8BFQ8|PDDC1_MOUSE & 1 & 2 & 2 & AIDFVDVTESNAR \\
\hline sp|Q8CFE2|CD027_MOUSE & 1 & 5 & 2 & DSPDELPVYVGTNEAK \\
\hline sp|Q8R1J3|ZCHC9_MOUSE & 1 & 2 & 2 & GM[147]SADYEDVLDVPK \\
\hline sp|Q8VDG7|PAFA2_MOUSE & 1 & 4 & 2 & TVVNVFPGGLDLM[147]TLK \\
\hline sp|Q8VDQ1|PTGR2_MOUSE & 1 & 4 & 2 & $\begin{array}{l}\text { GLENM[147]GVAFQSM[147]M[147]TGG } \\
\text { NVGK }\end{array}$ \\
\hline sp|Q91VE6|MK67I_MOUSE & 1 & 3 & 2 & GIDYSFPSLVLPK \\
\hline sp|Q91WE2|NIP30_MOUSE & 1 & 3 & 2 & GLDEDETNFLDEVSR \\
\hline sp|Q99LC2|CSTF1_MOUSE & 1 & 3 & 2 & LGM[147]ENDDTAVQYAIGR \\
\hline sp|Q9CQI9|MED30_MOUSE & 1 & 2 & 2 & IGQETVQDIVYR \\
\hline sp|Q9D753|EXOS8_MOUSE & 1 & 3 & 2 & ATTVNIGSISTADGSALVK \\
\hline sp|Q9D832|DNJB4_MOUSE & 1 & 5 & 2 & VIGYGLPFPK \\
\hline sp|Q9DBL1|ACDSB_MOUSE & 1 & 5 & 2 & SGNYYVLNGSK \\
\hline sp|Q9EPJ9|ARFG1_MOUSE & 1 & 3 & 2 & IFDDVSSGVSQLASK \\
\hline sp|Q9JK38|GNA1_MOUSE & 1 & 2 & 2 & VLGQLTETGVVSPEQFM[147]K \\
\hline sp|Q9WUN2|TBK1_MOUSE & 1 & 5 & 2 & LSSSQGTIESSLQDISSR \\
\hline sp|Q9Z2D8|MBD3_MOUSE & 1 & 2 & 2 & AFM[147]VTDDDIR \\
\hline sp|P18653|KS6A1_MOUSE & 0.9993 & 1 & 3 & DLKPENILLDEEGHIK \\
\hline sp|P97820|M4K4_MOUSE & 1 & 4 & 2 & GQNVLLTENAEVK \\
\hline sp|Q91YS8|KCC1A_MOUSE & 0.9999 & 2 & 2 & LIFQVLDAVK \\
\hline sp|Q921E2|RAB31_MOUSE & 0.9999 & 2 & 2 & GSAAAVIVYDITK \\
\hline sp|P0C7N9|PSMG4_MOUSE & 0.9998 & 2 & 2 & AAADADVSLHNFSAR \\
\hline sp|P59438|HPS5_MOUSE & 0.9998 & 4 & 2 & LLDPLVLFEPK \\
\hline sp|Q8C5Q4|GRSF1_MOUSE & 0.9998 & 2 & 2 & LGDEVDDVYLIR \\
\hline sp|Q9Z1B5|MD2L1_MOUSE & 0.9998 & 2 & 2 & YGLTLLTTTDPELIK \\
\hline sp|P47226|TES_MOUSE & 0.9997 & 2 & 2 & NVM[147]ILTNPVAAK \\
\hline sp|O09117|SYPL1_MOUSE & 0.9992 & 2 & 2 & SAFQINLNPLK \\
\hline sp|Q9D7H3|RTC1_MOUSE & 0.999 & 3 & 2 & AFVAGVLPLK \\
\hline sp|Q91X78|ERLN1_MOUSE & 0.9969 & 1 & 2 & ISEIEDAAFLAR \\
\hline sp|A6PWY4|WDR76_MOUSE & 1 & 3 & 2 & VFDSSSISSQLPLLSTIR \\
\hline sp|O08579|EMD_MOUSE & 1 & 3 & 2 & DYNDDYYEESYLTTK \\
\hline sp|O54784|DAPK3_MOUSE & 1 & 3 & 2 & ESLTEDEATQFLK \\
\hline sp|P33611|DPOA2_MOUSE & 1 & 3 & 2 & QLLSPSSFSPSATPSQK \\
\hline sp|P97789|XRN1_MOUSE & 1 & 4 & 2 & APELFSYIAK \\
\hline
\end{tabular}




\begin{tabular}{|c|c|c|c|c|}
\hline sp|Q3U1V6|UEVLD_MOUSE & 1 & 5 & 2 & DGVLSPSSQAQLSSR \\
\hline sp|Q64669|NQO1_MOUSE & 1 & 3 & 2 & ALIVLAHSEK \\
\hline sp|Q78JE5|FBX22_MOUSE & 1 & 2 & 2 & STYVLSNLAEVVER \\
\hline sp|Q810A3|TTC9C_MOUSE & 1 & 3 & 2 & AGVAFFHLQDYDR \\
\hline sp|Q8BKX1|BAIP2_MOUSE & 1 & 2 & 2 & EGDLITLLVPEAR \\
\hline sp|Q8BWR2|CA128_MOUSE & 1 & 2 & 2 & GLAYGLYLR \\
\hline sp|Q8C407|YIPF4_MOUSE & 1 & 3 & 2 & SAASLLVGEEFK \\
\hline sp|Q8CEC0|NUP88_MOUSE & 1 & 2 & 2 & GPSGGGVEPPLSQYQR \\
\hline sp|Q8CF89|TAB1_MOUSE & 1 & 3 & 2 & VLLQAFDVVER \\
\hline sp|Q8K194|SNR27_MOUSE & 1 & 3 & 2 & VDGSVNAYAINVSQK \\
\hline sp|Q8K409|DPOLB_MOUSE & 1 & 3 & 2 & EEM[147]LQM[147]QDIVLNEIK \\
\hline sp|Q8VDS4|RPR1A_MOUSE & 1 & 2 & 2 & SVYENDVLEQLK \\
\hline sp|Q8VDS8|STX18_MOUSE & 1 & 3 & 2 & TAVLDFVDDYLK \\
\hline sp|Q91WK1|SPRY4_MOUSE & 1 & 3 & 2 & VGLLLDYEAK \\
\hline sp|Q91XI1|DUS3L_MOUSE & 1 & 4 & 2 & ISEM[147]LLGPVPPGFVFLPK \\
\hline sp|Q9CR95|NECP1_MOUSE & 1 & 3 & 2 & ASGTGGLSLLPPPPGGK \\
\hline sp|Q9CY28|GTPB8_MOUSE & 1 & 3 & 2 & LFDPSLEDIGR \\
\hline sp|Q9CZX9|TMM85_MOUSE & 1 & 2 & 2 & GSGQGDSLYPVGYLDK \\
\hline sp|Q9D8M4|RL7L_MOUSE & 1 & 4 & 2 & TVPLTDNTVIEEHLGR \\
\hline sp|Q9DBX2|PHLP_MOUSE & 1 & 3 & 2 & EVLVLTSVR \\
\hline sp|Q9ER73|ELP4_MOUSE & 1 & 4 & 2 & NLSDTVVGLESFIGSER \\
\hline sp|Q9JLB2|MPP5_MOUSE & 1 & 4 & 2 & EGDEDNQPLAGLVPGK \\
\hline sp|Q9R099|TBL2_MOUSE & 1 & 3 & 2 & APIINIGIADTGK \\
\hline sp|Q9WVB0|RBPMS_MOUSE & 1 & 3 & 2 & ENTPSEANLQEEEVR \\
\hline sp|Q6ZPF4|FMNL3_MOUSE & 0.9983 & 1 & 2 & AAAVSLENVLLDVK \\
\hline sp|Q921Y2|IMP3_MOUSE & 0.9999 & 2 & 2 & VGPDVVTDPAFLVTR \\
\hline sp|Q3V009|TMED1_MOUSE & 0.9998 & 2 & 2 & SIQM[147]LTLLR \\
\hline sp|Q9CQ71|RFA3_MOUSE & 0.9991 & 2 & 2 & M[147]FILSDGEGK \\
\hline sp|Q9CQG2|MET10_MOUSE & 0.9983 & 2 & 2 & APEDVILALEER \\
\hline sp|O55125|NIPS1_MOUSE & 0.9904 & 1 & 2 & AGPNIYELR \\
\hline sp|O09114|PTGDS_MOUSE & 1 & 3 & 2 & AQGLTEEDIVFLPQPDK \\
\hline sp|P27601|GNA13_MOUSE & 1 & 4 & 2 & ALWEDSGIQNAYDR \\
\hline sp|P42669|PURA_MOUSE & 1 & 2 & 2 & GPGLGSTQGQTIALPAQGLIEFR \\
\hline sp|P62748|HPCL1_MOUSE & 1 & 3 & 2 & IYANFFPYGDASK \\
\hline sp|P97493|THIOM_MOUSE & 1 & 2 & 2 & TTFNVQDGPDFQDR \\
\hline sp|Q3U0V2|TRADD_MOUSE & 1 & 3 & 2 & DPALDSLAYEYER \\
\hline sp|Q3UE37|UBE2Z_MOUSE & 1 & 3 & 2 & GHFDYQSLLM[147]R \\
\hline sp|Q5SSK3|CQ042_MOUSE & 1 & 2 & 2 & LENLIDVPLIQYK \\
\hline sp|Q62086|PON2_MOUSE & 1 & 4 & 2 & FQEEENSLLHLK \\
\hline sp|Q8BJZ4|RT35_MOUSE & 1 & 3 & 2 & IPNFLHLTPVAIK \\
\hline sp|Q8BKF1|RPOM_MOUSE & 1 & 4 & 2 & QLAELLVQAVQM[147]PR \\
\hline sp|Q8BMZ5|SEN34_MOUSE & 1 & 3 & 2 & FGGDFLVYPGDPLR \\
\hline sp|Q8BNV1|TRM2A_MOUSE & 1 & 4 & 2 & DDLFTSEIFK \\
\hline sp|Q8BP40|PPA6_MOUSE & 1 & 4 & 2 & AAISQPGISEDLEK \\
\hline sp|Q8BVI5|STX16_MOUSE & 1 & 2 & 2 & QIVQSISDLNEIFR \\
\hline sp|Q8K2M0|RM38_MOUSE & 1 & 4 & 2 & TPPLGPM[147]PNEDIDVSNLER \\
\hline sp|Q8R322|GLE1_MOUSE & 1 & 4 & 2 & IEAITSSGQM[147]GSFIR \\
\hline
\end{tabular}




\begin{tabular}{|c|c|c|c|c|}
\hline sp|Q8R4Y8|RTTN_MOUSE & 1 & 5 & 2 & AILLYLLQGR \\
\hline sp|Q8VD00|TMM97_MOUSE & 1 & 3 & 2 & DPLM[147]QEPPVWFK \\
\hline sp|Q91VJ5|PQBP1_MOUSE & 1 & 3 & 2 & KDEELDPM[147]DPSSYSDAPR \\
\hline sp|Q920R0|ALS2_MOUSE & 1 & 5 & 2 & GTSDFPLYGGGSSVQR \\
\hline sp|Q921T2|TOIP1_MOUSE & 1 & 3 & 2 & FESLPAGSTLIFYK \\
\hline sp|Q9CXR1|DHRS7_MOUSE & 1 & 3 & 2 & LM[147]LISM[147]ANDLK \\
\hline sp|Q9CYY7|SLMO2_MOUSE & 1 & 2 & 2 & LNAEIEELAASAR \\
\hline sp|Q9CZU4|ERAL_MOUSE & 1 & 3 & 2 & ETQVILLDTPGIISPVK \\
\hline sp|Q9D2R8|RT33_MOUSE & 1 & 2 & 2 & VVSLFSEQPLAK \\
\hline sp|Q9EP72|CO024_MOUSE & 1 & 3 & 2 & SEVPGAAAEGPGGSGVGLGDR \\
\hline sp|Q9WV03|FA50A_MOUSE & 1 & 3 & 2 & SGPLFNFDVHDDVR \\
\hline sp|Q925E7|2ABD_MOUSE & 0.9951 & 1 & 2 & INLWHLEITDR \\
\hline sp|Q9JI46|NUDT3_MOUSE & 1 & 2 & 2 & LVGIFENQER \\
\hline sp|Q3UA16|SPC25_MOUSE & 0.9999 & 3 & 2 & LQFIFTSIDPK \\
\hline sp|Q6PAQ4|REXO4_MOUSE & 0.9999 & 3 & 2 & VSIVNQYGK \\
\hline sp|Q810J8|ZFYV1_MOUSE & 0.9999 & 3 & 2 & FLGDASEAYLK \\
\hline sp|Q8BH66|ATLA1_MOUSE & 0.9999 & 4 & 2 & SFLM[147]DFM[147]LR \\
\hline sp|Q8JZV7|NAGA_MOUSE & 0.9999 & 3 & 2 & ATEDVGSGVALVAR \\
\hline sp|Q9D7X8|GGCT_MOUSE & 0.9999 & 2 & 2 & SYLM[147]TNYESAPPSPQYK \\
\hline sp|O88952|LIN7C_MOUSE & 0.9999 & 2 & 2 & TEEGLGFNIM[147]GGK \\
\hline sp|Q9EQS3|MYCBP_MOUSE & 0.9998 & 2 & 2 & LVQYEPPQEEK \\
\hline sp|Q9WV84|NDKM_MOUSE & 0.9996 & 2 & 2 & M[147]LQAPESILAEHYR \\
\hline sp|Q9CQX4|PAF_MOUSE & 0.9995 & 2 & 2 & VLGSSTFVTNSSSSSR \\
\hline sp|Q9D4J1|EFHD1_MOUSE & 0.9987 & 1 & 2 & VFNPYTEFPEFSR \\
\hline sp|Q8R035|ICT1_MOUSE & 0.9975 & 2 & 2 & AGELVLTSESSR \\
\hline sp|Q5BL07|PEX1_MOUSE & 0.9963 & 1 & 2 & YPELFANLPIR \\
\hline sp|O35448|PPT2_MOUSE & 0.9929 & 2 & 2 & ESLRPLWEQVQGFR \\
\hline sp|Q99JF5|ERG19_MOUSE & 0.9913 & 1 & 2 & GLQVAPVLLSDELK \\
\hline sp|Q9CXA2|PRCM_MOUSE & 0.9913 & 1 & 2 & DLVDAASALTGAVK \\
\hline sp|A2ADY9|DDI2_MOUSE & 1 & 2 & 2 & NPPLAEALLSGDLEK \\
\hline sp|A2RSX7|CB060_MOUSE & 1 & 2 & 2 & DAQYLYLSGSK \\
\hline sp|A3KMP2|TTC38_MOUSE & 1 & 2 & 2 & VLELLLPIR \\
\hline sp|O35295|PURB_MOUSE & 1 & 2 & 2 & GGGGGGGGGPGGFQPAPR \\
\hline sp|O35623|BET1_MOUSE & 1 & 2 & 2 & LLAEM[147]DSQFDSTTGFLGK \\
\hline sp|Q03958|PFD6_MOUSE & 1 & 2 & 2 & ETLAQLQQEFQR \\
\hline sp|Q3UGP9|LRC58_MOUSE & 1 & 2 & 2 & DLTYDPPTLLELAAR \\
\hline sp|Q4FK66|PR38A_MOUSE & 1 & 2 & 2 & YVLEEAEQLEPR \\
\hline sp|Q4FZF3|DDX49_MOUSE & 1 & 2 & 2 & ELAYQIAEQFR \\
\hline sp|Q56A08|GPKOW_MOUSE & 1 & 3 & 2 & AVVVLSGPYR \\
\hline sp|Q61823|PDCD4_MOUSE & 1 & 3 & 2 & DLPELALDTPR \\
\hline sp|Q6P3D0|NUD16_MOUSE & 1 & 2 & 2 & EQLLEALQDLK \\
\hline sp|Q6PCP5|MFF_MOUSE & 1 & 3 & 2 & IQYEM[147]EYTEGISQR \\
\hline sp|Q80TH2|LAP2_MOUSE & 1 & 4 & 2 & IYDILGDDGPQPPSAAVK \\
\hline sp|Q80UW2|FBX2_MOUSE & 1 & 2 & 2 & TDAGSLYELTVR \\
\hline sp|Q8BGU5|CCNY_MOUSE & 1 & 2 & 2 & IVLGAILLASK \\
\hline sp|Q8BHL8|PSMF1_MOUSE & 1 & 2 & 2 & VLIDPSSGLPNR \\
\hline sp|Q8C163|EXOG_MOUSE & 1 & 2 & 2 & SPESTEPLALGAFVVPNK \\
\hline
\end{tabular}




\begin{tabular}{|c|c|c|c|c|}
\hline sp|Q8JZM0|TFB1M_MOUSE & 1 & 2 & 2 & FIPGLQM[147]LSDAAPGK \\
\hline sp|Q8K3J1|NDUS8_MOUSE & 1 & 2 & 2 & GLGM[147]TLSYLFR \\
\hline sp|Q8R3Y8|I2BP1_MOUSE & 1 & 2 & 2 & LALPSPALEYTLGSR \\
\hline sp|Q8VC70|RBMS2_MOUSE & 1 & 3 & 2 & GYGFVDFDSPSSAQK \\
\hline sp|Q8VCN9|TBCC_MOUSE & 1 & 2 & 2 & QGQAALAQLQAVLTER \\
\hline sp|Q8VHR5|P66B_MOUSE & 1 & 3 & 2 & LQQQAALSPTTAPAVSSVSK \\
\hline sp|Q8WTY4|CPIN1_MOUSE & 1 & 2 & 2 & KPNFEVGSSSQLK \\
\hline sp|Q91YY4|ATPF2_MOUSE & 1 & 2 & 2 & LTVEQAVLLSR \\
\hline sp|Q920Q8|NS1BP_MOUSE & 1 & 3 & 2 & LYIVGGSDPYGQK \\
\hline sp|Q922Q9|CHID1_MOUSE & 1 & 3 & 2 & FTQISPVWLQLK \\
\hline sp|Q99K43|PRC1_MOUSE & 1 & 2 & 2 & EIWELIGIPEEQR \\
\hline sp|Q99N94|RM09_MOUSE & 1 & 2 & 2 & LLSQGLAVYASPENR \\
\hline sp|Q9D1C1|UBE2C_MOUSE & 1 & 2 & 2 & LQQELM[147]ILM[147]TSGDK \\
\hline sp|Q9D920|L12R1_MOUSE & 1 & 2 & 2 & GLLSGQTSPTNAK \\
\hline sp|Q9DCT8|CRIP2_MOUSE & 1 & 2 & 2 & GVNTGAVGSYIYDKD \\
\hline sp|Q9JHE7|TSSC4_MOUSE & 1 & 2 & 2 & DAALAFLSSR \\
\hline sp|Q9JJZ4|UB2J1_MOUSE & 1 & 2 & 2 & TALLAIIGFM[147]PTK \\
\hline sp|Q9JKJ9|CP39A_MOUSE & 1 & 4 & 2 & TVLESISSVFGTAGK \\
\hline sp|Q9JLJ0|LITAF_MOUSE & 1 & 2 & 2 & GM[147]NPPSYYTQPVPVPNAN \\
\hline sp|Q9JMG1|EDF1_MOUSE & 1 & 2 & 2 & INEKPQVIADYESGR \\
\hline sp|P34056|AP2A_MOUSE & 1 & 3 & 2 & AVAEFLNR \\
\hline sp|P36993|PPM1B_MOUSE & 1 & 3 & 2 & GPTEQLVSPEPEVYEIVR \\
\hline sp|Q9JHD1|PCAF_MOUSE & 1 & 3 & 2 & LFMADLQR \\
\hline sp|P42230|STA5A_MOUSE & 0.9999 & 2 & 2 & IQAQFAQLGQLNPQER \\
\hline sp|Q3UX61|ARD1B_MOUSE & 0.9999 & 2 & 2 & YVSLHVR \\
\hline sp|Q80WC1|K2030_MOUSE & 0.9999 & 3 & 2 & VVPTLPEGLPVLLEK \\
\hline sp|Q91YD9|WASL_MOUSE & 0.9999 & 2 & 2 & FYGSQVNNISHTK \\
\hline sp|Q9DCS3|MECR_MOUSE & 0.9999 & 3 & 2 & DLGADYVLTEEELR \\
\hline sp|Q02614|S30BP_MOUSE & 0.9998 & 2 & 2 & DPQELVASFSER \\
\hline sp|Q8BSQ9|PB1_MOUSE & 0.9998 & 3 & 2 & VVDDEIYYFR \\
\hline sp|Q9DAU1|CNPY3_MOUSE & 0.9998 & 2 & 2 & ELGGLGEDANAEEEEGVQK \\
\hline sp|Q7TQK4|EXOS3_MOUSE & 0.9996 & 2 & 2 & LYPLEIVFGM[147]NGR \\
\hline sp|Q8K4F5|ABHDB_MOUSE & 0.9996 & 2 & 2 & LNLDTLAQHLDK \\
\hline sp|Q9D6Y7|MSRA_MOUSE & 0.9996 & 3 & 2 & VISAEEALPGR \\
\hline sp|A2APY7|CT007_MOUSE & 0.9994 & 2 & 2 & IFQTDIAEHALK \\
\hline sp|O70126|AURKB_MOUSE & 0.9994 & 3 & 2 & FGNVYLAR \\
\hline sp|Q60759|GCDH_MOUSE & 0.9994 & 2 & 2 & AITGIQAFTVGK \\
\hline sp|P49586|PCY1A_MOUSE & 0.9992 & 2 & 2 & TEGISTSDIITR \\
\hline sp|Q9Z0M5|LICH_MOUSE & 0.9992 & 2 & 2 & LYDEIISLM[147]K \\
\hline sp|Q9QYL7|ABT1_MOUSE & 0.9991 & 2 & 2 & NLLSAYGEVGR \\
\hline sp|O88520|SHOC2_MOUSE & 0.999 & 2 & 2 & LVLTNNQLSTLPR \\
\hline sp|Q9CR09|UFC1_MOUSE & 0.999 & 3 & 3 & LKEEYQSLIR \\
\hline sp|Q9DCF9|SSRG_MOUSE & 0.9983 & 2 & 2 & QQSEEDLLLQDFSR \\
\hline sp|Q8BGX2|CS052_MOUSE & 0.9982 & 2 & 2 & ILDVGFVGR \\
\hline sp|Q9Z1R4|CF047_MOUSE & 0.9978 & 2 & 2 & AILDALGLR \\
\hline sp|Q9DBY1|SYVN1_MOUSE & 0.9977 & 3 & 2 & VHTFPLFAIR \\
\hline sp|Q9Z2G0|FEM1B_MOUSE & 0.9977 & 2 & 2 & VLTLAALLLNR \\
\hline
\end{tabular}




\begin{tabular}{|c|c|c|c|c|}
\hline sp|P62484|ABI2_MOUSE & 0.996 & 1 & 2 & ALFDSYTNLER \\
\hline sp|A2A4P0|DHX8_MOUSE & 0.9957 & 1 & 2 & FSQYFYEAPIFTIPGR \\
\hline sp|Q9D735|CS043_MOUSE & 0.9913 & 1 & 2 & GGPGPTLSFVGK \\
\hline sp|Q3UBX0|TM109_MOUSE & 0.9904 & 1 & 2 & ETSADILTQIGR \\
\hline sp|O35972|RM23_MOUSE & 1 & 2 & 2 & NYLEQIYNVPVAAVR \\
\hline sp|Q2TPA8|HSDL2_MOUSE & 1 & 2 & 2 & ADVVM[147]SM[147]ATDDFVK \\
\hline sp|Q80VL1|TDRKH_MOUSE & 1 & 2 & 2 & DM[147]ATETDDSLASILTETK \\
\hline sp|Q8C156|CND2_MOUSE & 1 & 2 & 2 & TIEQNLSNINVSEADGK \\
\hline sp|Q8CHT0|AL4A1_MOUSE & 1 & 3 & 2 & TVIQAEIDAAAELIDFFR \\
\hline sp|Q8K2L8|TTC15_MOUSE & 1 & 2 & 2 & QVLNASSVEQSFVGLK \\
\hline sp|Q8R2N2|CIR1A_MOUSE & 1 & 2 & 2 & TDGTVEIYNLSANYFQEK \\
\hline sp|Q8VE18|CQ071_MOUSE & 1 & 2 & 2 & AANYDFYQLLEEK \\
\hline sp|Q91ZR2|SNX18_MOUSE & 1 & 2 & 2 & APEPGPPADGGPGAPAR \\
\hline sp|Q921W4|QORL1_MOUSE & 1 & 2 & 3 & LSAGVFRPLLDEPIPLYEAK \\
\hline sp|Q99MZ7|PECR_MOUSE & 1 & 2 & 2 & NQVAVVTGGGTGIGK \\
\hline sp|Q9D7A8|ARMC1_MOUSE & 1 & 2 & 2 & AEALASAIASTK \\
\hline sp|Q9DC71|RT15_MOUSE & 1 & 3 & 2 & TLEAQIIALTVR \\
\hline sp|Q9ESP1|SDF2L_MOUSE & 1 & 2 & 2 & AM[147]EGIFIKPGADLSTGHDEL \\
\hline sp|Q9WV85|NDK3_MOUSE & 1 & 2 & 2 & ALIGATDPGDAM[147]PGTIR \\
\hline sp|Q3V300|KIF22_MOUSE & 0.9999 & 3 & 2 & ALM[147]DLLQLAR \\
\hline sp|Q8BHE8|CB047_MOUSE & 0.9999 & 2 & 2 & QLLSASYEFQR \\
\hline sp|Q8BVU5|NUDT9_MOUSE & 0.9999 & 3 & 2 & EFGEEALNSLQK \\
\hline sp|Q8R107|PRLD1_MOUSE & 0.9999 & 2 & 2 & AVQEFGLAR \\
\hline sp|Q9CRA5|GOLP3_MOUSE & 0.9999 & 2 & 2 & SDAPTGDVLLDEALK \\
\hline sp|Q9D125|RT25_MOUSE & 0.9999 & 2 & 2 & IM[147]TVNYNTYGELGEGAR \\
\hline sp|Q9D2E2|TOE1_MOUSE & 0.9999 & 3 & 2 & VPVVDVQSDNFK \\
\hline sp|Q9DB90|CS061_MOUSE & 0.9999 & 2 & 2 & GGNQTSGIDFFITQER \\
\hline sp|Q9WVM3|APC7_MOUSE & 0.9999 & 2 & 2 & AIQLNSNSVQALLLK \\
\hline sp|Q9Z2E1|MBD2_MOUSE & 0.9999 & 2 & 2 & LQGLSASDVTEQIIK \\
\hline sp|Q8K0C9|GMDS_MOUSE & 0.9998 & 2 & 2 & FYQASTSELYGK \\
\hline sp|Q9ESW8|PGPI_MOUSE & 0.9998 & 2 & 2 & SAFVHVPPLGK \\
\hline sp|Q62036|AZI1_MOUSE & 0.9997 & 2 & 2 & PAEPTDFLM[147]LFEGSTSGR \\
\hline sp|Q99N87|RT05_MOUSE & 0.9997 & 3 & 2 & ЕРEPEPEVPDTK \\
\hline sp|Q9CYI4|LUC7L_MOUSE & 0.9997 & 2 & 2 & ALLDQLM[147]GTAR \\
\hline sp|Q9R1Z7|PTPS_MOUSE & 0.9997 & 2 & 3 & LHSPSLSDEENLR \\
\hline sp|Q64437|ADH7_MOUSE & 0.9997 & 3 & 2 & M[147]LTYDPM[147]LLFTGR \\
\hline sp|Q924W5|SMC6_MOUSE & 0.9996 & 2 & 2 & SAVLTALIVGLGGK \\
\hline sp|Q9R0X0|MED20_MOUSE & 0.9996 & 2 & 2 & SLQQTVELLTK \\
\hline sp|Q9D8V7|SC11C_MOUSE & 0.9993 & 2 & 2 & GDLLFLTNFR \\
\hline sp|Q9DB40|MED27_MOUSE & 0.9993 & 2 & 2 & TPLYSQLLQAYK \\
\hline sp|Q80W93|HYDIN_MOUSE & 0.9992 & 3 & 3 & QKLT[181]LLAQGQGLEPR \\
\hline sp|Q91Y86|MK08_MOUSE & 0.9988 & 3 & 2 & NIIGLLNVFTPQK \\
\hline sp|Q63810|CANB1_MOUSE & 0.9977 & 2 & 3 & M[147]M[147]VGNNLKDTQLQQIVDK \\
\hline sp|Q91ZF0|DJC24_MOUSE & 0.9973 & 2 & 2 & LILLYHPDK \\
\hline sp|Q8VC65|NRM_MOUSE & 0.996 & 2 & 2 & YFGVLQR \\
\hline sp|Q99KK9|SYHM_MOUSE & 0.9945 & 1 & 2 & DQGGELLSLR \\
\hline sp|Q9D6M3|GHC1_MOUSE & 0.9933 & 2 & 2 & SEGYFGM[147]YR \\
\hline
\end{tabular}




\begin{tabular}{|c|c|c|c|c|}
\hline sp|Q8BIJ7|RUFY1_MOUSE & 0.9902 & 2 & 2 & GSALQLQLSQLR \\
\hline sp|O89050|MKLN1_MOUSE & 1 & 3 & 2 & VFGGM[147]NEENM[147]TELLSSGLK \\
\hline sp|P04184|KITH_MOUSE & 1 & 2 & 2 & KLFASQQVLQYNSAN \\
\hline sp|P63271|SPT41_MOUSE & 1 & 2 & 3 & VSNFKPGVYAVSVTGR \\
\hline sp|Q8R088|GLP3L_MOUSE & 1 & 3 & 2 & DLVELDPEVEGTK \\
\hline sp|Q9D8T7|SLIRP_MOUSE & 1 & 3 & 2 & IPWTAAASELR \\
\hline sp|O70325|GPX41_MOUSE & 1 & 3 & 2 & TDVNYTQLVDLHAR \\
\hline sp|Q5SYD0|MYO1D_MOUSE & 0.9963 & 1 & 2 & LMYNSSNPVLK \\
\hline sp|P40338|VHL_MOUSE & 0.9999 & 3 & 2 & SLYEDLEDYPSVR \\
\hline sp|Q921G6|LRCH4_MOUSE & 0.9999 & 2 & 2 & SYDLSDITQADLSR \\
\hline sp|Q99JN2|KLH22_MOUSE & 0.9999 & 3 & 2 & LFVIGGSNNDAGYR \\
\hline sp|Q9D4J7|PHF6_MOUSE & 0.9999 & 3 & 2 & VAIDQQLTQQQLNGN \\
\hline sp|P15327|PMGE_MOUSE & 0.9998 & 3 & 2 & HYGALIGLNR \\
\hline sp|Q8K273|MMGT1_MOUSE & 0.9998 & 3 & 3 & NHPSFYVFNHR \\
\hline sp|Q8K3C3|LZIC_MOUSE & 0.9998 & 2 & 2 & KVEILTALR \\
\hline sp|A3KGB4|TBC8B_MOUSE & 0.9998 & 3 & 2 & DSLALWTFR \\
\hline sp|Q60967|PAPS1_MOUSE & 0.9996 & 2 & 2 & M[147]VAGANFYIVGR \\
\hline sp|Q8BZH4|POGZ_MOUSE & 0.9996 & 2 & 2 & SFLVASVLPGPDGNVNSPTR \\
\hline sp|Q9CYX7|RRP15_MOUSE & 0.9995 & 2 & 2 & GVVQLFNAVQK \\
\hline sp|P11930|NUD19_MOUSE & 0.9994 & 2 & 2 & LENFASLSALYR \\
\hline sp|Q6P6J9|TXD15_MOUSE & 0.9994 & 2 & 2 & GDPM[147]VVLSVVPGAAEDQR \\
\hline sp|Q9D1R1|T126B_MOUSE & 0.9994 & 2 & 2 & LFVTDALQSGDISK \\
\hline sp|Q80TF4|KLH13_MOUSE & 0.9993 & 3 & 2 & NFAALLSTGEFLK \\
\hline sp|Q8BJS4|UN84B_MOUSE & 0.9993 & 2 & 2 & ADVESQFPDWIR \\
\hline sp|Q3TMH2|SCRN3_MOUSE & 0.9991 & 2 & 2 & SPTFEPERPVAK \\
\hline sp|Q99KL7|RAB28_MOUSE & 0.9991 & 2 & 2 & ADIVNYNQEPLSR \\
\hline sp|Q8BP48|AMPM1_MOUSE & 0.9989 & 2 & 2 & EVLDIAAGM[147]IK \\
\hline sp|Q99M04|LIAS_MOUSE & 0.9985 & 3 & 2 & VGNELGFLYTASGPLVR \\
\hline sp|Q9CQT5|POMP_MOUSE & 0.9985 & 2 & 2 & NIQGLFAPLK \\
\hline sp|Q9CXZ1|NDUS4_MOUSE & 0.9984 & 2 & 2 & LDITTLTGVPEEHIK \\
\hline sp|O08738|CASP6_MOUSE & 0.9983 & 2 & 2 & IEIQTLTGLFK \\
\hline sp|P30280|CCND2_MOUSE & 0.9983 & 2 & 2 & SVEDPDQATTPTDVRDVDL \\
\hline sp|Q9Z0V7|TI17B_MOUSE & 0.9982 & 2 & 2 & EGSPAPGYPNYQQYH \\
\hline sp|Q8CFH6|SN1L2_MOUSE & 0.998 & 2 & 2 & IADFGFGNFFK \\
\hline sp|O08914|FAAH1_MOUSE & 0.9979 & 2 & 2 & LQSGELSPEAVLFTYLGK \\
\hline sp|P60766|CDC42_MOUSE & 0.9979 & 2 & 2 & TLGLFDTAGQEDYDR \\
\hline sp|Q9D6H2|HSB11_MOUSE & 0.9979 & 2 & 2 & DGYATFLR \\
\hline sp|Q9DD18|DTD1_MOUSE & 0.9979 & 2 & 2 & ASVTVGGEQISAIGR \\
\hline sp|Q3TKY6|SDC10_MOUSE & 0.9978 & 2 & 2 & EDQTLALLSQFK \\
\hline sp|Q9ERA0|TFCP2_MOUSE & 0.9978 & 2 & 2 & LFTNFSGADLLK \\
\hline sp|Q3TQB2|FXRD1_MOUSE & 0.9976 & 2 & 2 & TIDM[147]SPFLFTR \\
\hline sp|Q9D1H6|HRP20_MOUSE & 0.9976 & 2 & 2 & IAQEYYLELK \\
\hline sp|Q9D2X5|K0892_MOUSE & 0.9976 & 2 & 2 & GLFSFFQGR \\
\hline sp|Q9CQ02|COMD4_MOUSE & 0.9971 & 2 & 2 & ELLGQGIDYEK \\
\hline sp|Q80UU2|RPP38_MOUSE & 0.9969 & 1 & 2 & VPSLNVPWLPDR \\
\hline sp|Q9DBS5|KLC4_MOUSE & 0.9969 & 1 & 2 & DHPAVAATLNNLAVLYGK \\
\hline sp|P02468|LAMC1_MOUSE & 0.9966 & 1 & 2 & LSAEDLVLEGAGLR \\
\hline
\end{tabular}




\begin{tabular}{|c|c|c|c|c|}
\hline sp|Q64152|BTF3_MOUSE & 0.9963 & 2 & 2 & TATADDKKLQFSLK \\
\hline sp|Q8BRN9|C2D1B_MOUSE & 0.9963 & 1 & 2 & GM[147]NLPAPPGVTPDDLDAFVR \\
\hline sp|P19182|IFRD1_MOUSE & 0.9961 & 2 & 2 & VLYEFVLER \\
\hline sp|Q91Z38|TTC1_MOUSE & 0.9958 & 2 & 2 & AIQLNPTYIR \\
\hline sp|Q9D8T0|FAM3A_MOUSE & 0.9958 & 2 & 2 & IVSGAANVIGPK \\
\hline sp|O09111|NDUBB_MOUSE & 0.9954 & 2 & 2 & EVNGLPIM[147]ESNYFDPSK \\
\hline sp|Q9CYA0|CREL2_MOUSE & 0.9949 & 2 & 2 & YEFSEIR \\
\hline sp|Q6ZQF0|TOPB1_MOUSE & 0.9942 & 2 & 2 & NLTVALANSSR \\
\hline sp|Q8CI70|LRC20_MOUSE & 0.9933 & 2 & 2 & FM[147]TTFNQLR \\
\hline sp|Q7TSG2|CTDP1_MOUSE & 0.9931 & 2 & 2 & VLTQLVLSPDAPDR \\
\hline sp|Q8BRK8|AAPK2_MOUSE & 0.9923 & 1 & 2 & IADFGLSNM[147]M[147]SDGEFLR \\
\hline sp|O35459|ECH1_MOUSE & 0.9913 & 1 & 2 & EVDM[147]GLAADVGTLQR \\
\hline sp|P17095|HMGA1_MOUSE & 0.9913 & 1 & 2 & KQPPVSPGTALVGSQK \\
\hline sp|Q9CQ26|STABP_MOUSE & 0.9913 & 1 & 2 & NEFTITHVLIPR \\
\hline sp|Q9CRB2|NHP2_MOUSE & 0.9913 & 1 & 2 & ELLVNLNPIAQPLASR \\
\hline sp|Q9D0I4|STX17_MOUSE & 0.9913 & 1 & 2 & VAGIAAALGGGVLGFTGGK \\
\hline sp|Q8BW94|DYH3_MOUSE & 0.9908 & 2 & 2 & VFLEALNNNIR \\
\hline sp|P70353|NFYC_MOUSE & 0.9904 & 1 & 2 & M[147]ISAEAPVLFAK \\
\hline sp|P60762|MO4L1_MOUSE & 1 & 2 & 2 & VDPTVENEETFM[147]NR \\
\hline sp|Q8BLY7|HPS6_MOUSE & 1 & 2 & 2 & LLSDLSNFTGAAR \\
\hline sp|Q9DBZ1|IKIP_MOUSE & 1 & 2 & 2 & LLQTESSEFQGLQSK \\
\hline sp|Q8C863|ITCH_MOUSE & 0.9904 & 1 & 2 & FIDTGFSLPFYK \\
\hline sp|Q91W34|CP058_MOUSE & 0.9999 & 2 & 2 & ALVLETLNESR \\
\hline sp|Q4VAA7|SNX33_MOUSE & 0.9998 & 2 & 2 & IAETYSIEM[147]GPR \\
\hline sp|Q9DCT5|SDF2_MOUSE & 0.9997 & 2 & 2 & AM[147]EGIFM[147]KPSELLR \\
\hline sp|Q9D287|SPF27_MOUSE & 0.9996 & 2 & 2 & EAAAALVEEETR \\
\hline sp|Q9JKK1|STX6_MOUSE & 0.9995 & 2 & 2 & DQM[147]SASSVQALAER \\
\hline sp|P97434|MPRIP_MOUSE & 0.9993 & 2 & 2 & LLAEETAATISAIEAMK \\
\hline sp|Q3TRM4|PLPL6_MOUSE & 0.9993 & 3 & 2 & GDLIGVVEALTR \\
\hline sp|Q9D1I5|MCEE_MOUSE & 0.9988 & 2 & 3 & M[147]ELLHPLGSDSPITGFLQK \\
\hline sp|O55060|TPMT_MOUSE & 0.9983 & 2 & 2 & GALVAINPGDHDR \\
\hline sp|Q64362|AKTIP_MOUSE & 0.9983 & 2 & 2 & IDTTSPLNPEAAVLYEK \\
\hline sp|Q9Z2Q5|RM40_MOUSE & 0.9983 & 2 & 2 & VYTQVEFKR \\
\hline sp|Q3UH68|LIMC1_MOUSE & 0.9978 & 2 & 2 & TSVPESIASAGTGSPSK \\
\hline sp|Q8CE50|SNX30_MOUSE & 0.9976 & 2 & 2 & VEFDLPEYSVR \\
\hline sp|Q8BQZ4|K1219_MOUSE & 0.9973 & 2 & 2 & EVPVIFIHPLNTGLFR \\
\hline sp|Q99JY4|TRABD_MOUSE & 0.9971 & 2 & 2 & TVTQLVAEDGSR \\
\hline sp|Q8VCR3|CF035_MOUSE & 0.9966 & 1 & 2 & GTM[147]ATAALPESGSSLALR \\
\hline sp|Q9D892|ITPA_MOUSE & 0.9931 & 3 & 2 & IDLPEYQGEPDEISIQK \\
\hline sp|A2RTL5|RSRC2_MOUSE & 0.9913 & 1 & 2 & NTAM[147]DAQEALAR \\
\hline sp|O54916|REPS1_MOUSE & 0.9913 & 1 & 2 & LVAVAQSGFPLR \\
\hline sp|Q8BKW4|ZCHC4_MOUSE & 0.9913 & 1 & 2 & YLSFIQLPLAQR \\
\hline sp|Q8BU04|UBR7_MOUSE & 0.9913 & 1 & 2 & EDIQQFFEEFQSK \\
\hline sp|Q9CXK8|NIP7_MOUSE & 0.9913 & 1 & 2 & LHVTALDYLAPYAK \\
\hline sp|Q9D8Z1|ASCC1_MOUSE & 0.9913 & 1 & 2 & TFENFYFGSLR \\
\hline sp|Q9ET47|ESPN_MOUSE & 0.9913 & 1 & 2 & SFNM[147]M[147]SPTGDNSELLAEIK \\
\hline sp|Q9JI44|DMAP1_MOUSE & 0.9913 & 1 & 2 & TPEQVAEEEYLLQELR \\
\hline
\end{tabular}




\begin{tabular}{|c|c|c|c|c|}
\hline sp|Q9WU81|SPX2_MOUSE & 0.9913 & 1 & 2 & EQGPEPEAISFLGALR \\
\hline sp|P55302|AMRP_MOUSE & 0.9913 & 1 & 2 & IQEYNVLLDTLSR \\
\hline sp|Q5SS80|DHR13_MOUSE & 0.991 & 3 & 2 & LLT[181]HLLLPR \\
\hline sp|Q8BGI5|PEX26_MOUSE & 0.9904 & 1 & 2 & LSEAEELAVR \\
\hline sp|O35144|TERF2_MOUSE & 0.9977 & 2 & 2 & ALSTAQDSEAAFAK \\
\hline sp|Q80X71|T106B_MOUSE & 0.997 & 2 & 2 & GQENQLVALIPYSDQR \\
\hline sp|Q8C0M9|ASGL1_MOUSE & 0.9967 & 2 & 2 & GLGGLILVNK \\
\hline sp|Q69ZW3|EHBP1_MOUSE & 0.9954 & 2 & 3 & PK[142]PTSPNNLVNTVQEGETER \\
\hline sp|P70428|EXT2_MOUSE & 0.9914 & 1 & 2 & YVDDAGVPVSSAISR \\
\hline sp|P70445|4EBP2_MOUSE & 0.9913 & 1 & 2 & HAVGDEAQFEM[147]DI \\
\hline sp|Q61827|MAFK_MOUSE & 0.9913 & 1 & 2 & EAGENAPVLSDDELVSM[147]SVR \\
\hline sp|Q9WTK2|CDYL_MOUSE & 0.9913 & 1 & 2 & EVQSALSTAAADDSK \\
\hline sp|P55937|GOGA3_MOUSE & 0.9913 & 1 & 2 & VADAAASLEQQLEQVK \\
\hline sp|O35516|NOTC2_MOUSE & 0.9904 & 1 & 2 & EPLPPIVTFQLIPK \\
\hline sp|P61205|ARF3_MOUSE & 1 & 2 & 2 & DAVLLVFANK \\
\hline sp|Q61781|K1C14_MOUSE & 1 & 2 & 2 & VLDELTLAR \\
\hline sp|Q9Z1K7|APC2_MOUSE & 0.9993 & 2 & 2 & LPVS[167]IPAPQR \\
\hline sp|P09041|PGK2_MOUSE & 0.9961 & 1 & 3 & VSHVSTGGGASLELLEGK \\
\hline sp|P62309|RUXG_MOUSE & 0.9961 & 1 & 2 & GNSIIMLEALER \\
\hline sp|Q66JX5|FR1OP_MOUSE & 0.9961 & 1 & 2 & DLLVQTLENSGVLNR \\
\hline sp|P62746|RHOB_MOUSE & 0.9942 & 1 & 2 & QVELALWDTAGQEDYDR \\
\hline sp|Q9D1M7|FKB11_MOUSE & 0.9942 & 1 & 2 & DPLVIELGQK \\
\hline sp|Q61474|MSI1H_MOUSE & 0.9914 & 1 & 2 & GFGFVTFMDQAGVDK \\
\hline
\end{tabular}

Table S2

\begin{tabular}{lcccl}
\hline \multicolumn{1}{c}{ protein } & $\begin{array}{c}\text { protein } \\
\text { probability }\end{array}$ & $\begin{array}{c}\text { Num } \\
\text { unique peps }\end{array}$ & $\begin{array}{c}\text { precursor ion } \\
\text { charge }\end{array}$ & \multicolumn{1}{c}{ peptide sequence } \\
\hline sp|P68033|ACTC_MOUSE & 1 & 9 & 2 & AGFAGDDAPR \\
sp|Q64524|H2B2E_MOUSE & 1 & 10 & 2 & AMGIMNSFVNDIFER \\
sp|Q7TPR4|ACTN1_MOUSE & 1 & 35 & 2 & AGTQIENIEEDFR \\
sp|Q9WU78|PDC6I_MOUSE & 1 & 33 & 2 & ATLVKPTPVNVPVSQK \\
sp|Q62448|IF4G2_MOUSE & 1 & 23 & 2 & EWLTELFQQSK \\
sp|Q6PIC6|AT1A3_MOUSE & 0.9951 & 4 & 2 & GVGIISEGNETVEDIAAR \\
sp|P16110|LEG3_MOUSE & 1 & 13 & 2 & IQVLVEADHFK \\
sp|Q61206|PA1B2_MOUSE & 1 & 2 & 2 & IIVLGLLPR \\
sp|Q61941|NNTM_MOUSE & 1 & 11 & 2 & AVVLAANHFGR \\
sp|Q8BH74|NU107_MOUSE & 1 & 13 & 2 & AIYAALSGNLK \\
sp|Q9Z2I0|LETM1_MOUSE & 1 & 9 & 2 & ADDKLISEEGVDSLTVK \\
sp|Q921N6|DDX27_MOUSE & 1 & 13 & 2 & ALQEFDLALR \\
sp|Q6PAR5|GAPD1_MOUSE & 1 & 12 & 2 & AVETPPM[147]SSVNLLEGLSR \\
sp|Q9DBY8|NVL_MOUSE & 1 & 13 & 2 & AVANESGLNFISVK \\
sp|Q9WVL3|S12A7_MOUSE & 1 & 13 & 2 & DLQMFLYHLR \\
sp|P28271|ACOC_MOUSE & 1 & 10 & 2 & TSLSPGSGVVTYYLR \\
sp|Q9QZ21|AFAD_MOUSE & 1 & 12 & 2 & LAAGDQLLSVDGR \\
sp|Q80TP3|UBR5_MOUSE & 1 & 12 & LLTATNLVTLPNSR \\
\hline
\end{tabular}




\begin{tabular}{|c|c|c|c|c|}
\hline sp|Q8VED5|K2C79_MOUSE & 0.9908 & 1 & 3 & NKYEDEINKR \\
\hline sp|P42859|HD_MOUSE & 1 & 14 & 2 & TLFGTNLASQFDGLSSNPSK \\
\hline sp|P43247|MSH2_MOUSE & 1 & 11 & 2 & DSLIIIDELGR \\
\hline sp|Q6P5H2|NEST_MOUSE & 1 & 12 & 2 & AGLELEQEVVGLEDPR \\
\hline sp|Q64310|SURF4_MOUSE & 1 & 9 & 2 & GQNDLM[147]GTAEDFADQFLR \\
\hline sp|Q60790|RASA3_MOUSE & 1 & 11 & 2 & FGDEFLGELR \\
\hline sp|Q69ZN7|MYOF_MOUSE & 1 & 9 & 2 & ANVTVLDTQIR \\
\hline sp|Q8BTI8|SRRM2_MOUSE & 1 & 11 & 2 & IPAASAAAM[147]NLASAR \\
\hline sp|Q921G8|GCP2_MOUSE & 1 & 11 & 2 & ILPVAASYSTVTR \\
\hline sp|Q6P2K6|P4R3A_MOUSE & 1 & 9 & 2 & AESDGSLLLESK \\
\hline sp|Q91ZU6|BPA1_MOUSE & 1 & 15 & 2 & AGNDLIESSEGEEASNLQYK \\
\hline sp|Q3TLH4|BA2D1_MOUSE & 1 & 9 & 2 & ESVTDYTTPSSSLPNTVATNNAK \\
\hline sp|Q99K01|PDXD1_MOUSE & 1 & 11 & 2 & AVPVSNIAPAAVGR \\
\hline sp|P48722|HS74L_MOUSE & 1 & 11 & 2 & EDINSIEIVGGATR \\
\hline sp|Q07113|MPRI_MOUSE & 1 & 8 & 2 & LASM[147]QLDYR \\
\hline sp|Q62383|SPT6H_MOUSE & 1 & 9 & 2 & IDTASLGDSTDSYIEVLDGSR \\
\hline sp|Q6P549|SHIP2_MOUSE & 1 & 10 & 2 & ALQDM[147]SSTAPPAPLQPSIR \\
\hline sp|Q8R0Y6|FTHFD_MOUSE & 1 & 7 & 2 & ANATEFGLASGVFTR \\
\hline sp|Q8VHE0|SEC63_MOUSE & 1 & 10 & 2 & LIM[147]VLAGASEFDPQYNK \\
\hline sp|Q3TZZ7|ESYT2_MOUSE & 1 & 7 & 2 & ALALLEDEEQAVR \\
\hline sp|Q9CY27|GPSN2_MOUSE & 1 & 8 & 2 & LPVGTTATLYFR \\
\hline sp|P53986|MOT1_MOUSE & 1 & 4 & 2 & AAQSPQQHSSGDPTEEESPV \\
\hline sp|Q6Q899|DDX58_MOUSE & 1 & 9 & 2 & DNVAELEQVVYKPQK \\
\hline sp|Q9DBT5|AMPD2_MOUSE & 1 & 10 & 2 & SAPYEFPEESPIEQLEER \\
\hline sp|P46425|GSTP2_MOUSE & 0.9995 & 1 & 2 & AFLSSPEHVNRPINGNGK \\
\hline sp|Q9EPK7|XPO7_MOUSE & 1 & 9 & 2 & AALSGSYVNFGVFR \\
\hline sp|Q9ERA6|TFP11_MOUSE & 1 & 7 & 2 & AVSSNVGAYM[147]QPGAR \\
\hline sp|P97386|DNLI3_MOUSE & 1 & 7 & 2 & HVLDALDPNAYEAFK \\
\hline sp|Q8BI84|MIA3_MOUSE & 1 & 6 & 2 & ELEGLLEDMSIR \\
\hline sp|Q9D2M8|UB2V2_MOUSE & 0.9998 & 6 & 2 & WTGM[147]IIGPPR \\
\hline sp|Q61703|ITIH2_MOUSE & 1 & 3 & 2 & IQPSGGTNINEALLR \\
\hline sp|Q8BH24|TM9S4_MOUSE & 1 & 6 & 2 & ITEEYYVHLIADNLPVATR \\
\hline sp|Q91YR7|PRP6_MOUSE & 1 & 6 & 2 & LSQVSDSVSGQTVVDPK \\
\hline sp|Q9ERG2|STRN3_MOUSE & 1 & 6 & 2 & AYIASAGADALAK \\
\hline sp|Q9CR68|UCRI_MOUSE & 0.9999 & 2 & 2 & LQVTNVLSQPLTQATVK \\
\hline sp|P08775|RPB1_MOUSE & 1 & 5 & 2 & INISQVIAVVGQQNVEGK \\
\hline sp|Q61263|SOAT1_MOUSE & 1 & 6 & 2 & LLAAEAEELKPLFM[147]K \\
\hline sp|Q8BPM2|M4K5_MOUSE & 1 & 7 & 2 & LISENTEGSAQAPQLPR \\
\hline sp|Q99KD5|UN45A_MOUSE & 1 & 3 & 2 & ASFITANGVSLLK \\
\hline sp|Q9D0A3|CO038_MOUSE & 1 & 6 & 2 & VNTGFLM[147]SSYK \\
\hline sp|Q9DBC3|FTSJ2_MOUSE & 1 & 8 & 2 & IHAFVQDTTLSEPR \\
\hline sp|O35678|MGLL_MOUSE & 1 & 3 & 2 & GAYLLM[147]ESSR \\
\hline sp|Q8BX70|VP13C_MOUSE & 1 & 7 & 2 & SLDVFNIILVR \\
\hline sp|Q8BYW9|AER61_MOUSE & 1 & 5 & 2 & AFTDYDVIHLK \\
\hline sp|Q924Z4|LASS2_MOUSE & 1 & 5 & 2 & AGTLIM[147]ALHDASDYLLESAK \\
\hline
\end{tabular}




\begin{tabular}{|c|c|c|c|c|}
\hline sp|Q9Z160|COG1_MOUSE & 1 & 8 & 2 & ALQLLYDLR \\
\hline sp|O08759|UBE3A_MOUSE & 1 & 6 & 2 & M[147]M[147]ETFQQLITYK \\
\hline sp|P22892|AP1G1_MOUSE & 1 & 6 & 2 & AM[147]ELSFALVNGNNIR \\
\hline sp|Q52KI8|SRRM1_MOUSE & 1 & 6 & 2 & DSSVQEATSTSDILK \\
\hline sp|Q6PGH2|HN1L_MOUSE & 1 & 4 & 2 & GSGIFDESTPVQTR \\
\hline sp|Q80U72|LAP4_MOUSE & 1 & 7 & 2 & SLEELLLDANQLR \\
\hline sp|Q8BVG4|DPP9_MOUSE & 1 & 4 & 2 & ELVQPFSSLFPK \\
\hline sp|Q8R3S6|EXOC1_MOUSE & 1 & 6 & 2 & ALQEGDLVSSR \\
\hline sp|Q9D4H1|EXOC2_MOUSE & 1 & 6 & 2 & ASNTADTLFQEVLGR \\
\hline sp|O54988|SLK_MOUSE & 1 & 6 & 2 & AGNILFTLDGDIK \\
\hline sp|P16056|MET_MOUSE & 1 & 5 & 2 & GDLTIANLGTSEGR \\
\hline sp|O35382|EXOC4_MOUSE & 1 & 5 & 2 & FIQEIEHALGLGPAK \\
\hline sp|P39053|DYN1_MOUSE & 1 & 4 & 2 & SSVLENFVGR \\
\hline sp|P39447|ZO1_MOUSE & 1 & 5 & 2 & LAGGNDVGIFVAGVLEDSPAAK \\
\hline sp|Q7TSC1|BAT2_MOUSE & 1 & 5 & 2 & AVGTPGANAGGAGPGISAM[147]SR \\
\hline sp|Q8BU03|PWP2_MOUSE & 1 & 3 & 2 & AGQLLPVVQFLQK \\
\hline sp|Q8BYH7|TBC17_MOUSE & 1 & 5 & 2 & IFSGGLSPGLR \\
\hline sp|Q8CCB4|VPS53_MOUSE & 1 & 6 & 2 & M[147]VLLDLPSIGSQVVR \\
\hline sp|Q8K368|FANCI_MOUSE & 1 & 6 & 2 & FVSDLLTALFR \\
\hline sp|Q8R5L3|VPS39_MOUSE & 1 & 8 & 2 & AINLLPANTQINDIR \\
\hline sp|Q99NH0|ANR17_MOUSE & 1 & 6 & 2 & NVSDYTPLSLAASGGYVNIIK \\
\hline sp|Q9DC40|TELO2_MOUSE & 1 & 6 & 2 & LLGDLPDELLEAR \\
\hline sp|Q9QX47|SON_MOUSE & 1 & 5 & 2 & AGIEGPLLASEVER \\
\hline sp|Q9R0L6|PCM1_MOUSE & 1 & 7 & 2 & ALYALQDIVSR \\
\hline sp|P83741|WNK1_MOUSE & 1 & 5 & 2 & IGDLGLATLK \\
\hline sp|Q8CJG0|I2C2_MOUSE & 1 & 5 & 2 & YAQGADSVEPM[147]FR \\
\hline sp|P17897|LYZ1_MOUSE & 0.9976 & 2 & 2 & STDYGIFQINSR \\
\hline sp|P03911|NU4M_MOUSE & 1 & 5 & 2 & IILPSLM[147]LLPLTWLSSPK \\
\hline sp|P45377|ALD2_MOUSE & 1 & 4 & 3 & AVQREDLFIVSK \\
\hline sp|P97452|BOP1_MOUSE & 1 & 4 & 2 & VNVDPEDLIPK \\
\hline sp|Q3U487|HECD3_MOUSE & 1 & 4 & 2 & AGLPLPAALAFVPR \\
\hline sp|Q6PF93|PK3C3_MOUSE & 1 & 5 & 2 & DGDESSPILTSFELVK \\
\hline sp|Q6PR54|RIF1_MOUSE & 1 & 7 & 2 & TIGDLSTLTASEIK \\
\hline sp|Q8BIG7|CMTD1_MOUSE & 1 & 2 & 2 & PGGVLAVLR \\
\hline sp|Q8BUV3|GEPH_MOUSE & 1 & 5 & 2 & DLVQDPSLLGGTISAYK \\
\hline sp|Q8VBZ3|CLPT1_MOUSE & 1 & 4 & 2 & NLLTGETEADPEM[147]IK \\
\hline sp|Q9D8V0|HM13_MOUSE & 1 & 3 & 2 & QYQLLFTQGSGENK \\
\hline sp|Q9WV70|NOC2L_MOUSE & 1 & 3 & 2 & SIAFPELVLPTVLQLK \\
\hline sp|Q99KH8|STK24_MOUSE & 1 & 7 & 2 & KTSYLTELIDR \\
\hline sp|P01027|CO3_MOUSE & 0.9999 & 3 & 2 & SSVAVPYVIVPLK \\
\hline sp|Q8BIV3|RNBP6_MOUSE & 0.9985 & 2 & 2 & EGFVEYTEQVVK \\
\hline sp|O54827|AT10A_MOUSE & 0.9919 & 2 & 2 & ASPSPSLVIDGR \\
\hline sp|O35604|NPC1_MOUSE & 1 & 5 & 2 & LQEETLDQQLGR \\
\hline sp|O70579|PM34_MOUSE & 1 & 4 & 2 & LSSLDVFIIGAIAK \\
\hline sp|P58021|TM9S2_MOUSE & 1 & 5 & 2 & RPSENLGQVLFGER \\
\hline
\end{tabular}




\begin{tabular}{|c|c|c|c|c|}
\hline sp|Q3UDW8|HGNAT_MOUSE & 1 & 2 & 2 & ADPLSADYQPETR \\
\hline sp|Q61464|ZN638_MOUSE & 1 & 6 & 2 & DVTVLSM[147]AEEQDLQQER \\
\hline sp|Q6PIP5|NUDC1_MOUSE & 1 & 6 & 2 & LPTDVTAYDNR \\
\hline sp|Q8VDF2|UHRF1_MOUSE & 1 & 6 & 2 & YDDYPEHGVDIVK \\
\hline sp|Q91V04|TRAM1_MOUSE & 1 & 3 & 2 & LDFSTGNFNVLAVR \\
\hline sp|Q9DBF1|AL7A1_MOUSE & 1 & 5 & 2 & GAPTTSLVSVAVTK \\
\hline sp|Q9QY36|ARD1A_MOUSE & 1 & 4 & 2 & YYADGEDAYAM[147]K \\
\hline sp|Q91W97|HKDC1_MOUSE & 0.9956 & 2 & 2 & M[147]ISGLYM[147]GELVR \\
\hline sp|P31786|ACBP_MOUSE & 1 & 5 & 2 & TYVEKVDELKK \\
\hline sp|P41216|ACSL1_MOUSE & 1 & 2 & 2 & LM[147]ITGAAPVSATVLTFLR \\
\hline sp|P58404|STRN4_MOUSE & 1 & 5 & 2 & ALIASAGADALAK \\
\hline sp|Q0VGY8|TANC1_MOUSE & 1 & 7 & 2 & ANFQEIISALPFVK \\
\hline sp|Q3TCJ1|F175B_MOUSE & 1 & 3 & 2 & AIYQVYNALQEK \\
\hline sp|Q3UVK0|ERMP1_MOUSE & 1 & 5 & 2 & AFINLEAAGVGGK \\
\hline sp|Q5H8C4|VP13A_MOUSE & 1 & 4 & 2 & ALVGGAVGGLAGAASK \\
\hline sp|Q60953|PML_MOUSE & 1 & 4 & 2 & DNSVSSFLDSTR \\
\hline sp|Q6P9Q6|FKB15_MOUSE & 1 & 5 & 2 & EVATDGLLQGNSR \\
\hline sp|Q80Y81|RNZ2_MOUSE & 1 & 4 & 2 & LDNIFLTR \\
\hline sp|Q8C8R3|ANK2_MOUSE & 1 & 3 & 2 & VVTEEVTTTTTTITEK \\
\hline sp|Q8K1X1|BRWD2_MOUSE & 1 & 3 & 2 & LLLDPDFSLLQR \\
\hline sp|Q8R4G6|MGT5A_MOUSE & 1 & 4 & 2 & TLAVLLDNILQR \\
\hline sp|Q8VDC0|SYLM_MOUSE & 1 & 4 & 2 & AM[147]QDALADLPEWYGIK \\
\hline sp|Q91W86|VPS11_MOUSE & 1 & 3 & 2 & GNYPVTGLAFR \\
\hline sp|Q99PG2|OGFR_MOUSE & 1 & 3 & 2 & QSALDYFLFAVR \\
\hline sp|Q9EPU4|CPSF1_MOUSE & 1 & 4 & 2 & VLVDSSFGQPTTQGEVR \\
\hline sp|Q9ERG0|LIMA1_MOUSE & 1 & 5 & 2 & SQDVGFWEGEVVR \\
\hline sp|Q9ET30|TM9S3_MOUSE & 1 & 4 & 2 & DAFVYAIK \\
\hline sp|Q9JIX8|ACINU_MOUSE & 1 & 4 & 2 & KVTLGDTLTR \\
\hline sp|P46467|VPS4B_MOUSE & 1 & 4 & 2 & GILLFGPPGTGK \\
\hline sp|Q922W5|P5CR1_MOUSE & 0.9999 & 2 & 2 & LGAQALLGAAK \\
\hline sp|O35609|SCAM3_MOUSE & 1 & 3 & 2 & AQQEFAAGVFSNPAVR \\
\hline sp|P11688|ITA5_MOUSE & 1 & 3 & 2 & VTAPLEAEYSGLVR \\
\hline sp|P81117|NUCB2_MOUSE & 1 & 3 & 2 & AATADLEQYDR \\
\hline sp|Q0KL02|TRIO_MOUSE & 1 & 5 & 2 & DNFDAFYSEVAELGR \\
\hline sp|Q3TMX7|QSOX2_MOUSE & 1 & 5 & 2 & EGSDAVWLLDSGSVR \\
\hline sp|Q3U186|SYRM_MOUSE & 1 & 2 & 2 & GGVTFLEDVLNEVQSR \\
\hline sp|Q3UMF0|COBL1_MOUSE & 1 & 3 & 2 & DYQAQEPLDLTK \\
\hline sp|Q6ZQ93|UBP34_MOUSE & 1 & 10 & 2 & SFLLLAASTLLK \\
\hline sp|Q8BTY8|SCFD2_MOUSE & 1 & 3 & 2 & SQIAVNDVFM[147]ALR \\
\hline sp|Q8BXN9|TM87A_MOUSE & 1 & 3 & 2 & ADEIESYLENLK \\
\hline sp|Q8C0L8|COG5_MOUSE & 1 & 3 & 2 & GALEAYVQSVR \\
\hline sp|Q8C754|VPS52_MOUSE & 1 & 3 & 2 & FLEQLQELDAK \\
\hline sp|Q8CBQ5|P4K2B_MOUSE & 1 & 3 & 2 & IAAIDNGLAFPFK \\
\hline sp|Q8K2C9|PTAD1_MOUSE & 1 & 3 & 3 & VELSDVQNPAISITDNVLHFK \\
\hline sp|Q8R0A0|T2FB_MOUSE & 1 & 4 & 3 & VVTTNYKPVANHQYNIEYER \\
\hline
\end{tabular}




\begin{tabular}{|c|c|c|c|c|}
\hline sp|Q8R5J9|PRAF3_MOUSE & 1 & 2 & 2 & TPM[147]GIILDALEQQEDNINK \\
\hline sp|Q8VEL2|MTMRE_MOUSE & 1 & 2 & 2 & AATPSPSGAIGGLLEQFAR \\
\hline sp|Q922U1|PRPF3_MOUSE & 1 & 5 & 2 & LFEAVEEGR \\
\hline sp|Q99LB7|SARDH_MOUSE & 1 & 3 & 2 & DPSGGPVSLDFVK \\
\hline sp|Q9CQJ6|DENR_MOUSE & 1 & 3 & 2 & QETGITEGQGPVGEEEEK \\
\hline sp|Q9D853|METLA_MOUSE & 1 & 3 & 2 & SGNTVAALVFQK \\
\hline sp|Q9WV68|DECR2_MOUSE & 1 & 5 & 2 & VAFITGGGSGIGFR \\
\hline sp|Q61411|RASH_MOUSE & 1 & 3 & 2 & LVVVGAGGVGK \\
\hline sp|Q3UV17|K22O_MOUSE & 0.99 & 1 & 2 & AQYEDIAQK \\
\hline sp|Q8K0V4|CNOT3_MOUSE & 1 & 3 & 2 & M[147]LDNDSILVDAIR \\
\hline sp|Q921C5|BICD2_MOUSE & 1 & 4 & 2 & VGLLATLQDTQK \\
\hline sp|Q811J3|IREB2_MOUSE & 0.9999 & 3 & 2 & EGIPLIILAGK \\
\hline sp|P00397|COX1_MOUSE & 0.9983 & 2 & 2 & M[147]IGAPDM[147]AFPR \\
\hline sp|Q8BZ98|DYN3_MOUSE & 0.9964 & 2 & 2 & SSVLENFVGR \\
\hline sp|Q9ERY9|ERG28_MOUSE & 0.9955 & 2 & 2 & YLEAEPVSR \\
\hline sp|O08784|TCOF_MOUSE & 1 & 3 & 2 & AGAVTSSASLSSPALAK \\
\hline sp|O35657|NEUR1_MOUSE & 1 & 3 & 2 & GTLLAFAEAR \\
\hline sp|P03888|NU1M_MOUSE & 1 & 3 & 2 & GPNIVGPYGILQPFADAM[147]K \\
\hline sp|P70206|PLXA1_MOUSE & 1 & 4 & 2 & FVDDLFETIFSTAHR \\
\hline sp|Q3UMC0|SPAT5_MOUSE & 1 & 6 & 2 & ALANESGLNFLAIK \\
\hline sp|Q5DTT3|CJ018_MOUSE & 1 & 4 & 2 & VIPILPALSYALLEAK \\
\hline sp|Q5RJG1|NOL10_MOUSE & 1 & 3 & 2 & DLENLGLTHLIGSPFLR \\
\hline sp|Q68FF6|GIT1_MOUSE & 1 & 3 & 2 & SLSSPTDNLELSAR \\
\hline sp|Q80YV3|TRRAP_MOUSE & 1 & 5 & 2 & NFIQTILTSLIEK \\
\hline sp|Q8BM72|HSP13_MOUSE & 1 & 4 & 2 & IFTPEELEAEVGR \\
\hline sp|Q8R151|ZNFX1_MOUSE & 1 & 5 & 2 & INVFDFGQWPSK \\
\hline sp|Q8VCR7|ABHEB_MOUSE & 1 & 3 & 2 & FSVLLLHGIR \\
\hline sp|Q91X52|DCXR_MOUSE & 1 & 4 & 2 & GVPGAIVNVSSQASQR \\
\hline sp|Q9D8N2|FAM45_MOUSE & 1 & 3 & 2 & M[147]M[147]ESYIAVLTK \\
\hline sp|Q9QWT9|KIFC1_MOUSE & 1 & 4 & 2 & LTYLLQNSLGGSAK \\
\hline sp|Q9Z2G6|SE1L1_MOUSE & 1 & 3 & 2 & AADM[147]GNPVGQSGLGM[147]AYLYGR \\
\hline sp|P10648|GSTA2_MOUSE & 1 & 3 & 2 & SHGQDYLVGNR \\
\hline sp|Q3B7Z2|OSBP1_MOUSE & 1 & 4 & 2 & IPM[147]PVNFNEPLSM[147]LQR \\
\hline sp|Q8BLR2|CPNE4_MOUSE & 0.9999 & 2 & 2 & DIVQFVPFR \\
\hline sp|P28028|BRAF1_MOUSE & 0.9999 & 3 & 2 & M[147]LNVTAPTPQQLQAFK \\
\hline sp|Q8K4L0|DDX54_MOUSE & 0.9997 & 3 & 2 & AGLTEPVLIR \\
\hline sp|Q6GQT1|A2MP_MOUSE & 0.9992 & 3 & 2 & M[147]VSGFIPLKPTVK \\
\hline sp|Q8BFR1|ZCCHL_MOUSE & 0.9989 & 2 & 2 & FLLQEVELR \\
\hline sp|O35621|PMM1_MOUSE & 0.994 & 1 & 2 & NGM[147]LNVSPIGR \\
\hline sp|Q9CZX7|TM55A_MOUSE & 0.9917 & 2 & 2 & ISSVGSALPR \\
\hline sp|O88487|DC1I2_MOUSE & 1 & 3 & 2 & EAAVSVQEESDLEK \\
\hline sp|P52875|TM165_MOUSE & 1 & 3 & 2 & M[147]SPDEGQEELEEVQAELK \\
\hline sp|P97478|COQ7_MOUSE & 1 & 2 & 2 & IYAGQM[147]AVLGR \\
\hline sp|Q2HXL6|EDEM3_MOUSE & 1 & 3 & 2 & FTGATIFEEYAR \\
\hline sp|Q3THK3|T2FA_MOUSE & 1 & 3 & 2 & IYQEEEM[147]PESGAGSEFNR \\
\hline
\end{tabular}




\begin{tabular}{|c|c|c|c|c|}
\hline sp|Q3UHJ0|AAK1_MOUSE & 1 & 4 & 2 & AGQTQPNPGILPIQPALTPR \\
\hline sp|Q60596|XRCC1_MOUSE & 1 & 3 & 2 & HFFLYGEFPGDER \\
\hline sp|Q60855|RIPK1_MOUSE & 1 & 3 & 2 & AEYNEVLLEEGK \\
\hline sp|Q80TE0|RPAP1_MOUSE & 1 & 4 & 2 & VSSLLLPVPK \\
\hline sp|Q80U70|SUZ12_MOUSE & 1 & 5 & 2 & ETLTTELQTR \\
\hline sp|Q811D0|DLG1_MOUSE & 1 & 3 & 2 & QVTPDGESDEVGVIPSK \\
\hline sp|Q8BI72|CARF_MOUSE & 1 & 4 & 2 & GSASFVSSLLK \\
\hline sp|Q8BM55|TM214_MOUSE & 1 & 4 & 2 & SQSVFTGNPSVWLK \\
\hline sp|Q8BT07|CEP55_MOUSE & 1 & 5 & 2 & YSSSSLFEQLEEK \\
\hline sp|Q8BZ20|PAR12_MOUSE & 1 & 4 & 2 & LGLSSDLVSR \\
\hline sp|Q8CGU1|CACO1_MOUSE & 1 & 3 & 2 & AALLGEELASAAGAR \\
\hline sp|Q8K0Q5|RHG18_MOUSE & 1 & 4 & 2 & IEEGSLETEGLLR \\
\hline sp|Q8R2M2|TDIF2_MOUSE & 1 & 3 & 2 & LTSSSIDPGLNIK \\
\hline sp|Q8VIM9|IRGQ_MOUSE & 1 & 3 & 2 & PLPQGDVTALFLGPPGSGK \\
\hline sp|Q91VW5|GOGA4_MOUSE & 1 & 3 & 2 & SLLEELASQLDSR \\
\hline sp|Q91WR3|ASCC2_MOUSE & 1 & 3 & 2 & HNIFQNDEFDVFSR \\
\hline sp|Q91YK2|RRP1B_MOUSE & 1 & 3 & 2 & LGALPDSSSDLPVQK \\
\hline sp|Q99J27|ACATN_MOUSE & 1 & 4 & 2 & YTAGPQPLNIFYK \\
\hline sp|Q99KB8|GLO2_MOUSE & 1 & 3 & 2 & HVEPGNAAIQEK \\
\hline sp|Q99LB2|DHRS4_MOUSE & 1 & 4 & 2 & LAEDGAHVVVSSR \\
\hline sp|Q99M28|RNPS1_MOUSE & 1 & 3 & 2 & DHIMEIFSTYGK \\
\hline sp|Q9D2N9|VP33A_MOUSE & 1 & 4 & 2 & IISAAFEER \\
\hline sp|Q9DBR0|AKAP8_MOUSE & 1 & 3 & 2 & TVEFLQEYIINR \\
\hline sp|Q9JJA2|COG8_MOUSE & 1 & 3 & 2 & ISQFLQVLETDLYR \\
\hline sp|Q9WTR1|TRPV2_MOUSE & 1 & 3 & 2 & GVPEELTGLLEYLR \\
\hline sp|Q9Z2V5|HDAC6_MOUSE & 1 & 3 & 2 & LVDALM[147]GAEIR \\
\hline sp|Q64261|CDK6_MOUSE & 0.9917 & 1 & 2 & VQTSEEGM[147]PLSTIR \\
\hline sp|P63011|RAB3A_MOUSE & 1 & 2 & 2 & M[147]SESLDTADPAVTGAK \\
\hline sp|Q04692|SMRCD_MOUSE & 0.9999 & 2 & 2 & QEQLYSGLFNR \\
\hline sp|Q3U308|CP084_MOUSE & 0.9999 & 3 & 2 & DLPSLDPLPPYVLAEAQLR \\
\hline sp|Q8VHE6|DYH5_MOUSE & 0.9999 & 7 & 2 & RTDLNYIAAVDLK \\
\hline sp|P24638|PPAL_MOUSE & 0.9998 & 2 & 2 & LQGGVLLAQILK \\
\hline sp|Q8BZQ7|ANC2_MOUSE & 0.9998 & 2 & 2 & IEELFSIIR \\
\hline sp|Q99KR7|PPIF_MOUSE & 0.9998 & 3 & 2 & HVGPGVLSM[147]AN \\
\hline sp|P83510|TNIK_MOUSE & 0.9993 & 2 & 2 & NIATYYGAFIK \\
\hline sp|P62858|RS28_MOUSE & 0.9984 & 2 & 2 & EGDVLTLLESER \\
\hline sp|Q8R2U4|ME11A_MOUSE & 0.9978 & 2 & 2 & TAGLSLLAEER \\
\hline sp|P45878|FKBP2_MOUSE & 0.9968 & 2 & 2 & LVIPSELGYGER \\
\hline sp|P08032|SPTA1_MOUSE & 0.9967 & 3 & 2 & FLTLLAK \\
\hline sp|Q8C7B8|ZSWM4_MOUSE & 0.9943 & 2 & 2 & LQPALTSR \\
\hline sp|O35682|MYADM_MOUSE & 1 & 2 & 2 & TTITTTTSSSTTVGSAR \\
\hline sp|O70481|UBR1_MOUSE & 1 & 2 & 2 & INSENAEALAQLLTLAR \\
\hline sp|O88746|TOM1_MOUSE & 1 & 4 & 2 & SSPDLTGVVAVYEDLR \\
\hline sp|P48771|CX7A2_MOUSE & 1 & 2 & 2 & LFQEDNGM[147]PVHLK \\
\hline sp|P62313|LSM6_MOUSE & 1 & 2 & 2 & GNNVLYISTQK \\
\hline
\end{tabular}




\begin{tabular}{|c|c|c|c|c|}
\hline sp|P97314|CSRP2_MOUSE & 1 & 2 & 2 & GFGYGQGAGALVHAQ \\
\hline sp|Q00547|HMMR_MOUSE & 1 & 3 & 2 & DVTAQLESVQEK \\
\hline sp|Q3TVI8|PBIP1_MOUSE & 1 & 2 & 2 & QEGLALFGVELAPVR \\
\hline sp|Q3UVG3|F91A1_MOUSE & 1 & 3 & 2 & VQGDYFETLLYK \\
\hline sp|Q60772|CDN2C_MOUSE & 1 & 3 & 2 & DGTGFAVIHDAAR \\
\hline sp|Q64FW2|RETST_MOUSE & 1 & 3 & 2 & ATVQSVLLDSAGR \\
\hline sp|Q6DVA0|LEMD2_MOUSE & 1 & 3 & 2 & ELQALGFQPGPITDTTR \\
\hline sp|Q6NVE8|WDR44_MOUSE & 1 & 2 & 2 & LLASAGQDNIVR \\
\hline sp|Q6NZN0|RBM26_MOUSE & 1 & 3 & 2 & VIQPLVQQPILPVVK \\
\hline sp|Q80XU3|NUCKS_MOUSE & 1 & 2 & 3 & TPSPKEEDEEAESPPEKK \\
\hline sp|Q8BFW7|LPP_MOUSE & 1 & 3 & 2 & M[147]LYDM[147]ENPPADDYFGR \\
\hline sp|Q8BTZ5|ANR46_MOUSE & 1 & 2 & 2 & LLESLEEQEVK \\
\hline sp|Q8BUY5|CC001_MOUSE & 1 & 2 & 2 & AGAVAADSPGFVEDR \\
\hline sp|Q8C3I8|BRP16_MOUSE & 1 & 3 & 2 & DQGAYLILR \\
\hline sp|Q8C9B9|DIDO1_MOUSE & 1 & 3 & 2 & TASPLEHILQTLFGK \\
\hline sp|Q8K3X4|EAP1_MOUSE & 1 & 2 & 2 & YGLSAAAAAAAAAAAVEQR \\
\hline sp|Q8R307|VPS18_MOUSE & 1 & 3 & 2 & LGALQGDPDALTLYR \\
\hline sp|Q8R3P6|CO044_MOUSE & 1 & 3 & 2 & AALAFGFLDLLK \\
\hline sp|Q8R3V5|SHLB2_MOUSE & 1 & 2 & 2 & LASDAGIFFTR \\
\hline sp|Q8R5H6|WASF1_MOUSE & 1 & 3 & 2 & IENDVATILSR \\
\hline sp|Q8VD04|GRAP1_MOUSE & 1 & 4 & 2 & TQTGDSSSVSSFSYR \\
\hline sp|Q91VS7|MGST1_MOUSE & 1 & 2 & 2 & IYHTIAYLTPLPQPNR \\
\hline sp|Q91VX9|TM168_MOUSE & 1 & 2 & 2 & TVDIEEADPPQLGDFTR \\
\hline sp|Q91VY9|ZN622_MOUSE & 1 & 3 & 3 & VHSFFIPDIEYLSDLK \\
\hline sp|Q922S8|KIF2C_MOUSE & 1 & 4 & 2 & FSLVDLAGNER \\
\hline sp|Q922Y1|UBXN1_MOUSE & 1 & 2 & 2 & GEEPGQDQDPVQLLSGFPR \\
\hline sp|Q923D5|WBP11_MOUSE & 1 & 2 & 2 & AVSILPLLGHGVPR \\
\hline sp|Q9CQU3|RER1_MOUSE & 1 & 2 & 2 & LGQIYQSWLDK \\
\hline sp|Q9CYK1|SYWM_MOUSE & 1 & 3 & 2 & YGEFFPLPK \\
\hline sp|Q9D0M0|EXOS7_MOUSE & 1 & 3 & 3 & VYIVHGVQEDLR \\
\hline sp|Q9D1C8|VPS28_MOUSE & 1 & 2 & 2 & AM[147]DEIQPDLR \\
\hline sp|Q9DB96|NGDN_MOUSE & 1 & 2 & 2 & ASGASLQGHPAVLR \\
\hline sp|Q9DC29|ABCB6_MOUSE & 1 & 2 & 2 & APDIILLDEATSALDTSNER \\
\hline sp|Q9DCD2|SYF1_MOUSE & 1 & 2 & 2 & FYEDNGQLDDAR \\
\hline sp|Q9QUJ7|ACSL4_MOUSE & 1 & 4 & 2 & SDQSYVISFVVPNQK \\
\hline sp|Q9QWF0|CAF1A_MOUSE & 1 & 2 & 2 & LVGGQGPIDSFLR \\
\hline sp|Q9QY06|MYO9B_MOUSE & 1 & 3 & 2 & TPIESLFIEATER \\
\hline sp|Q9Z1T6|FYV1_MOUSE & 1 & 3 & 2 & DYFPEQIYWSPLLNK \\
\hline sp|Q9Z2A5|ATE1_MOUSE & 1 & 3 & 2 & SLEDLIFQSLPENASHK \\
\hline sp|Q9Z2L7|CRLF3_MOUSE & 1 & 2 & 2 & LIEHGVNTADDLVR \\
\hline sp|O35638|STAG2_MOUSE & 0.9999 & 2 & 2 & M[147]YSDAFLNDSYLK \\
\hline sp|P25425|PO2F1_MOUSE & 1 & 3 & 2 & LYGNDFSQTTISR \\
\hline sp|P42232|STA5B_MOUSE & 1 & 4 & 2 & IQAQFAQLGQLNPQER \\
\hline sp|P61620|S61A1_MOUSE & 1 & 5 & 3 & GM[147]EFEGAIIALFHLLATR \\
\hline sp|P84075|HPCA_MOUSE & 0.997 & 1 & 2 & IYANFFPYGDASK \\
\hline
\end{tabular}




\begin{tabular}{|c|c|c|c|c|}
\hline sp|P15116|CADH2_MOUSE & 0.9999 & 3 & 2 & LNGDFAQLNLK \\
\hline sp|P70700|RPA2_MOUSE & 0.9999 & 3 & 2 & ISLTIVDAVISPPSVPK \\
\hline sp|Q60928|GGT1_MOUSE & 0.9999 & 2 & 2 & FVDVSQVIR \\
\hline sp|Q8CI95|OSB11_MOUSE & 0.9999 & 3 & 2 & SVILHLLSQLK \\
\hline sp|Q91V01|PCAT3_MOUSE & 0.9999 & 2 & 2 & LATSLGASEQALR \\
\hline sp|Q9D0P8|RAYL_MOUSE & 0.9999 & 2 & 2 & SQTSGISLPGVLVGTK \\
\hline sp|Q9JJL8|SYSM_MOUSE & 0.9999 & 2 & 2 & VLIALLESNQQK \\
\hline sp|Q9QZ08|NAGK_MOUSE & 0.9999 & 2 & 2 & LGILTHLYR \\
\hline sp|Q02780|NFIA_MOUSE & 0.9999 & 2 & 2 & LDLVM[147]VILFK \\
\hline sp|Q91W69|EPN3_MOUSE & 0.9999 & 2 & 2 & NIVHNYSEAEIK \\
\hline sp|Q9CTG6|AT132_MOUSE & 0.9996 & 3 & 2 & ELSLLGLLVM[147]R \\
\hline sp|Q9R0Q9|MPU1_MOUSE & 0.9996 & 2 & 2 & GLGLGIVAGSLLVK \\
\hline sp|Q60848|HELLS_MOUSE & 0.9995 & 2 & 2 & LISQIQPEVNR \\
\hline sp|Q91ZN5|S35B2_MOUSE & 0.9994 & 2 & 2 & APDEVLLAPR \\
\hline sp|Q9WUU9|MCM3A_MOUSE & 0.9994 & 3 & 2 & LPLYLPQTLVSFPDSIK \\
\hline sp|P00848|ATP6_MOUSE & 0.9993 & 2 & 2 & LSM[147]AIPLWAGAVITGFR \\
\hline sp|Q8BTI7|ANR52_MOUSE & 0.9993 & 2 & 2 & DAVSPFSFSLLK \\
\hline sp|Q9JHS3|MAPIP_MOUSE & 0.9993 & 2 & 2 & NGNQAFNEDSLK \\
\hline sp|Q3TYS2|CQ062_MOUSE & 0.9991 & 2 & 2 & DIQDVNVEEEK \\
\hline sp|O88848|ARL6_MOUSE & 0.999 & 3 & 2 & IPILFFANK \\
\hline sp|Q80TZ9|RERE_MOUSE & 0.999 & 2 & 2 & VDSFFYILGYNPETR \\
\hline sp|P97412|LYST_MOUSE & 0.9988 & 5 & 2 & DLSGLLVSAFK \\
\hline sp|Q9CPP0|NPM3_MOUSE & 0.9988 & 2 & 2 & DHDNQEIAVPVANLR \\
\hline sp|Q8BFS6|CSTP1_MOUSE & 0.9986 & 2 & 2 & LTEQAVEAINK \\
\hline sp|Q8R1S0|COQ6_MOUSE & 0.9981 & 2 & 2 & ILLLEAGPK \\
\hline sp|Q8VHN7|GPR98_MOUSE & 0.998 & 4 & 2 & FAEPCVLR \\
\hline sp|Q9R0M6|RAB9A_MOUSE & 0.9976 & 2 & 2 & EPESFPFVILGNK \\
\hline sp|Q8BYU6|TOIP2_MOUSE & 0.9957 & 5 & 2 & FESLPAGSTLIFYK \\
\hline sp|Q9WTL7|LYPA2_MOUSE & 0.9956 & 2 & 2 & FGALTAEK \\
\hline sp|Q9Z2R6|U119A_MOUSE & 0.9949 & 1 & 2 & QPIGPEDVLGLQR \\
\hline sp|Q9CQT2|RBM7_MOUSE & 0.9919 & 2 & 2 & TLFVGNLETK \\
\hline sp|Q9CY57|CA077_MOUSE & 0.9917 & 1 & 2 & EQLDNQLDAYM[147]SK \\
\hline sp|P03930|ATP8_MOUSE & 1 & 2 & 2 & VSSQTFPLAPSPK \\
\hline sp|P24610|PAX3_MOUSE & 1 & 3 & 2 & TTFTAEQLEELER \\
\hline sp|P70302|STIM1_MOUSE & 1 & 3 & 2 & YAEEELEQVR \\
\hline sp|P70677|CASP3_MOUSE & 1 & 4 & 2 & LEFM[147]HILTR \\
\hline sp|Q09143|CTR1_MOUSE & 1 & 2 & 2 & VIYAM[147]AEDGLLFK \\
\hline sp|Q3UA37|QRIC1_MOUSE & 1 & 3 & 2 & EIQEAIAVANATTM[147]H \\
\hline sp|Q3UGY8|BIG3_MOUSE & 1 & 4 & 2 & NLIDTLSTPLTGR \\
\hline sp|Q3UW53|NIBAN_MOUSE & 1 & 4 & 2 & VLTSEEEYSLLSDK \\
\hline sp|Q64430|ATP7A_MOUSE & 1 & 5 & 2 & QIEAVGFPAFIK \\
\hline sp|Q8BHY8|SNX14_MOUSE & 1 & 3 & 2 & LVSLITLLR \\
\hline sp|Q8BTG3|T11L1_MOUSE & 1 & 3 & 2 & AIFSVLDLM[147]K \\
\hline sp|Q8C5W3|TBCEL_MOUSE & 1 & 3 & 2 & YYVDVPQEEVPFR \\
\hline sp|Q8CIM8|INT4_MOUSE & 1 & 3 & 2 & FLQEVDFFQR \\
\hline
\end{tabular}




\begin{tabular}{|c|c|c|c|c|}
\hline sp|Q91VZ6|SMAP1_MOUSE & 1 & 3 & 2 & LLYEANLPENFR \\
\hline sp|Q9JLV1|BAG3_MOUSE & 1 & 3 & 2 & ELLALDSVDPEGR \\
\hline sp|Q9QYC7|VKGC_MOUSE & 1 & 3 & 2 & DGLTGELGYLNPGVFTQSR \\
\hline sp|Q9QYH6|MAGD1_MOUSE & 1 & 3 & 2 & ATEAVLWEALR \\
\hline sp|Q9R0I7|YLPM1_MOUSE & 1 & 3 & 2 & VFSSEQGLGESSALSQSIIAAK \\
\hline sp|P61028|RAB8B_MOUSE & 0.9993 & 2 & 2 & SSTNVEEAFFTLAR \\
\hline sp|Q8JZR6|S4A8_MOUSE & 0.9984 & 2 & 2 & FLFILLGPVGK \\
\hline sp|Q9CQK7|RWDD1_MOUSE & 1 & 4 & 3 & AKFDAELLEIKK \\
\hline sp|P10922|H10_MOUSE & 0.9999 & 2 & 2 & YSDM[147]IVAAIQAEK \\
\hline sp|Q3UUQ7|PGAP1_MOUSE & 0.9998 & 3 & 2 & AFFDLIDADTK \\
\hline sp|Q8CHC4|SYNJ1_MOUSE & 0.9998 & 3 & 2 & VLDAYGLLGVLR \\
\hline sp|Q8K370|ACD10_MOUSE & 0.9998 & 4 & 2 & LSLQPSEAIFLDDLGSNLK \\
\hline sp|Q8BGZ2|F168A_MOUSE & 0.9997 & 2 & 2 & SIPSAIYPAPVAAPR \\
\hline sp|Q922H1|ANM3_MOUSE & 0.9997 & 3 & 2 & QTVFLLEKPFPVK \\
\hline sp|P58854|GCP3_MOUSE & 0.9996 & 3 & 2 & YLLLGQGDFIR \\
\hline sp|Q9CQZ6|NDUB3_MOUSE & 0.9996 & 3 & 2 & M[147]ELPDYR \\
\hline sp|P47713|PA24A_MOUSE & 0.9995 & 3 & 2 & DVPVVAILGSGGGFR \\
\hline sp|Q99JP7|GGT7_MOUSE & 0.9995 & 3 & 2 & LPEDEPAPAAPLR \\
\hline sp|O55242|OPRS1_MOUSE & 0.9994 & 2 & 2 & QYAGLDHELAFSR \\
\hline sp|Q8K5B2|MCFD2_MOUSE & 0.9994 & 2 & 2 & DDDKNNDGYIDYAEFAK \\
\hline sp|Q5U430|UBR3_MOUSE & 0.9992 & 3 & 2 & LDPDYFISSVFER \\
\hline sp|Q9QZH6|ECSIT_MOUSE & 0.9992 & 3 & 2 & IFVHYPR \\
\hline sp|Q9Z0J0|NPC2_MOUSE & 0.9991 & 2 & 2 & LPVKNEYPSIK \\
\hline sp|Q6P8H8|ALG8_MOUSE & 0.999 & 2 & 2 & AILLAILPM[147]SLLSVEK \\
\hline sp|Q8K4M5|COMD1_MOUSE & 0.999 & 2 & 2 & LSEVEESINR \\
\hline sp|Q9CQB5|CISD2_MOUSE & 0.9987 & 3 & 2 & QLPVPDSITGFAR \\
\hline sp|Q03173|ENAH_MOUSE & 0.9986 & 2 & 2 & VEDGSFPGGGNTGSVSLASSK \\
\hline sp|Q9EPN1|NBEA_MOUSE & 0.9984 & 3 & 2 & EISNFEYLM[147]FLNTIAGR \\
\hline sp|Q9R0Q4|MO4L2_MOUSE & 0.9983 & 2 & 2 & EYAVNEVVGGIK \\
\hline sp|Q8VCI5|PEX19_MOUSE & 0.9975 & 2 & 2 & ELAEEEPHLVEQFQK \\
\hline sp|Q80U78|PUM1_MOUSE & 0.9973 & 1 & 2 & SASSASSLFSPSSTLFSSSR \\
\hline sp|Q6ZPE2|MTMR5_MOUSE & 0.9964 & 2 & 2 & GLLALLFPLR \\
\hline sp|Q8CHT3|INT5_MOUSE & 0.9957 & 2 & 2 & EQPLLFELLK \\
\hline sp|Q91XL9|OSBL1_MOUSE & 0.9917 & 1 & 2 & ITM[147]PVIFNEPLSFLQR \\
\hline sp|Q80UG2|PLXA4_MOUSE & 0.9911 & 1 & 2 & FVDDLFETIFSTAHR \\
\hline sp|A2AAJ9|OBSCN_MOUSE & 0.9904 & 3 & 2 & EDENFVCIR \\
\hline sp|P97857|ATS1_MOUSE & 0.99 & 1 & 2 & GIGYFFVLQPK \\
\hline sp|O89017|LGMN_MOUSE & 1 & 2 & 2 & DYTGEDVTPENFLAVLR \\
\hline sp|Q3UFM5|NOM1_MOUSE & 1 & 3 & 2 & ELITEAQTQASGAGNK \\
\hline sp|Q5PRF0|HTR5A_MOUSE & 1 & 2 & 2 & VLILEQLLNSIK \\
\hline sp|Q5SUC9|SCO1_MOUSE & 1 & 2 & 2 & LVGLTGTKEEIDGVAR \\
\hline sp|Q64282|IFIT1_MOUSE & 1 & 3 & 2 & ISEQVQFLDIK \\
\hline sp|Q91WG2|RABE2_MOUSE & 1 & 2 & 2 & LQAELETSEQVQR \\
\hline sp|Q99MU3|DSRAD_MOUSE & 1 & 3 & 2 & YLNTNPVGGLLEYAR \\
\hline sp|Q9CR88|RT14_MOUSE & 1 & 2 & 2 & HLADHGLLSGVQR \\
\hline
\end{tabular}




\begin{tabular}{|c|c|c|c|c|}
\hline sp|Q9D187|FA96B_MOUSE & 1 & 2 & 2 & SGERPVTAGEEDEEVPDSIDAR \\
\hline sp|Q9Z0R9|FADS2_MOUSE & 1 & 3 & 2 & ALIDIVSSLK \\
\hline sp|Q61771|KIF3B_MOUSE & 1 & 2 & 2 & HLIIENFIPLEEK \\
\hline sp|O08601|MTP_MOUSE & 0.9999 & 3 & 2 & SGFTTANQVLGVSSK \\
\hline sp $|\mathrm{O} 35350| \mathrm{CAN} 1 \_M O U S E$ & 0.9999 & 2 & 2 & APSDLYQIILK \\
\hline sp|O88879|APAF_MOUSE & 0.9999 & 2 & 2 & GSPLVVSLIGALLR \\
\hline sp|Q3SXD3|HDDC2_MOUSE & 0.9999 & 2 & 2 & LQDFYDSTAGK \\
\hline sp|Q3U5F4|YRDC_MOUSE & 0.9999 & 2 & 2 & LPESEPVEAASPER \\
\hline sp|Q80XQ2|TBCD5_MOUSE & 0.9999 & 2 & 2 & TFPEM[147]QFFQQENVR \\
\hline sp|Q8BKX6|SMG1_MOUSE & 0.9999 & 4 & 2 & AQDTFQTIEGIIR \\
\hline sp|Q9CQ75|NDUA2_MOUSE & 0.9999 & 2 & 2 & TVSLNNLSADEVTR \\
\hline sp|Q9DBA9|TF2H1_MOUSE & 0.9999 & 2 & 2 & M[147]LQEDPVLFQLYK \\
\hline sp|Q9EQG9|C43BP_MOUSE & 0.9999 & 2 & 2 & DVLYLSAIR \\
\hline sp|Q3TEL6|RN157_MOUSE & 0.9999 & 2 & 2 & VSYLLQEIYGIENK \\
\hline sp|Q66T02|PKHG5_MOUSE & 0.9998 & 3 & 2 & SLGEVLLPVFER \\
\hline sp|Q8R080|GTSE1_MOUSE & 0.9998 & 2 & 2 & VPQFSVGESPGGVTPK \\
\hline sp|Q8VHL1|SETD7_MOUSE & 0.9998 & 2 & 2 & VYVADSLISSAGEGLFSK \\
\hline sp|Q99LH1|NOG2_MOUSE & 0.9998 & 2 & 2 & VIDSSDVVVQVLDAR \\
\hline sp|Q05793|PGBM_MOUSE & 0.9997 & 5 & 2 & VIPYFTQTPYSFLPLPTIK \\
\hline sp|Q3UGP8|AG10B_MOUSE & 0.9997 & 2 & 2 & YFILPYIIYR \\
\hline sp|Q9D4H2|GCC1_MOUSE & 0.9997 & 2 & 2 & TQLATLTSSLATVTQEK \\
\hline sp|Q9Z1X9|CC45L_MOUSE & 0.9997 & 2 & 2 & LQEFLADM[147]GLPLK \\
\hline sp|Q6IQX7|CHSS2_MOUSE & 0.9996 & 2 & 2 & LTVLLPLAAAER \\
\hline sp|Q6PEV3|WIPF2_MOUSE & 0.9996 & 2 & 2 & GSSGGYGPGAAALQPK \\
\hline sp|Q6XUX1|RIPK5_MOUSE & 0.9996 & 2 & 3 & SPLYGQLVDLGYLSSSHR \\
\hline sp|Q6ZWZ2|UB2R2_MOUSE & 0.9996 & 2 & 2 & FPIDYPYSPPTFR \\
\hline sp|P40201|CHD1_MOUSE & 0.9927 & 2 & 2 & M[147]LDILAEYLK \\
\hline sp|Q5SWT3|S2535_MOUSE & 0.9994 & 2 & 2 & LGTYGLAESR \\
\hline sp|Q91ZR1|RAB4B_MOUSE & 0.9994 & 2 & 2 & M[147]GSGIQYGDISLR \\
\hline sp|P46414|CDN1B_MOUSE & 0.9993 & 2 & 2 & NLFGPVNHEELTR \\
\hline sp|Q3U5Q7|CMPK2_MOUSE & 0.9993 & 2 & 2 & AFYSLGNYLVASEIAK \\
\hline sp|Q9CQ86|CQ037_MOUSE & 0.9993 & 2 & 2 & EEYPGIEIESR \\
\hline sp|P62311|LSM3_MOUSE & 0.9992 & 2 & 2 & GDGVVLVAPPLR \\
\hline sp|Q5BLK4|ZCHC6_MOUSE & 0.9992 & 2 & 2 & NTEPVGQLWLGLLR \\
\hline sp|Q8R550|SH3K1_MOUSE & 0.9992 & 2 & 2 & M[147]EPAVSSQAAIEELK \\
\hline sp|P54116|STOM_MOUSE & 0.9991 & 2 & 2 & EASM[147]VITESPAALQLR \\
\hline sp|Q501J2|F173A_MOUSE & 0.9991 & 2 & 2 & LQAELPVGAR \\
\hline sp|Q3UDR8|YIPF3_MOUSE & 0.999 & 2 & 2 & DIPAVLPAAR \\
\hline sp|Q9EPQ7|STAR5_MOUSE & 0.999 & 2 & 2 & SM[147]AEFYPNLQK \\
\hline sp|Q9CYZ6|CS060_MOUSE & 0.9989 & 2 & 2 & DAPIATLVQR \\
\hline sp|P28741|KIF3A_MOUSE & 0.9988 & 2 & 2 & SAKPETVIDSLLQ \\
\hline sp|Q9CYA6|ZCHC8_MOUSE & 0.9988 & 2 & 2 & LVNYPGFNISTPR \\
\hline sp|Q9R1S3|PIGN_MOUSE & 0.9988 & 2 & 2 & EATLPFLFTPFK \\
\hline sp|Q9R207|NBN_MOUSE & 0.9988 & 2 & 2 & LLPAAGAAPGEPYR \\
\hline sp|Q9JKX4|AATF_MOUSE & 0.9987 & 2 & 2 & ALLTTNQLPQPDVFPVFK \\
\hline
\end{tabular}




\begin{tabular}{|c|c|c|c|c|}
\hline sp|Q5KU39|VPS41_MOUSE & 0.9986 & 4 & 2 & IVLLM[147]DFDSEK \\
\hline sp|P0C1Q2|PDE11_MOUSE & 0.9985 & 2 & 2 & DISNDLDLTSLSYK \\
\hline sp|Q76KJ5|RPA34_MOUSE & 0.9985 & 2 & 2 & EATLLASSSEAGGR \\
\hline sp|Q9D850|TMM68_MOUSE & 0.9985 & 2 & 2 & TFLGDPIPYDPK \\
\hline sp|Q9R0D8|WDR54_MOUSE & 0.9985 & 2 & 2 & TISALDLAPEVGK \\
\hline sp|Q8K2F8|LS14A_MOUSE & 0.9981 & 2 & 2 & YEGILYTIDTENSTVALAK \\
\hline sp|Q9CPV5|PMF1_MOUSE & 0.9981 & 2 & 2 & NQELADAVLAGR \\
\hline sp|Q9DCI9|RM32_MOUSE & 0.998 & 2 & 2 & QQIGAQEGGPFR \\
\hline sp|Q9CQ18|RNH2C_MOUSE & 0.9978 & 2 & 2 & HDADGLQASFR \\
\hline sp|Q9D8Y1|T126A_MOUSE & 0.9978 & 2 & 2 & ILNVTQAR \\
\hline sp|Q9WTK3|GPAA1_MOUSE & 0.9976 & 2 & 2 & YGVEALTLR \\
\hline sp|P70444|BID_MOUSE & 0.9975 & 2 & 2 & IEPDSESQEEIIHNIAR \\
\hline sp|Q07139|ECT2_MOUSE & 0.9974 & 2 & 2 & LPSVALLLNDLK \\
\hline sp|Q8BMD6|CN101_MOUSE & 0.9974 & 3 & 2 & LPGTGIDPEVLLSEAIR \\
\hline sp|Q9D600|PSF2_MOUSE & 0.9974 & 2 & 2 & TNLQPSESTQSQDF \\
\hline sp|Q8BTJ4|ENPP4_MOUSE & 0.9973 & 2 & 2 & EVDDLIGDIVLK \\
\hline sp|Q8QZX2|CD015_MOUSE & 0.9973 & 2 & 2 & TNPM[147]VFLSQFPLGK \\
\hline sp|Q924L1|LTMD1_MOUSE & 0.9972 & 2 & 2 & LGIGQLTAQEVK \\
\hline sp|Q7TSZ8|NACC1_MOUSE & 0.9971 & 2 & 2 & FSTPDLALNR \\
\hline sp|Q61207|SAP_MOUSE & 0.997 & 2 & 2 & TVVTEAGNLLK \\
\hline sp|Q9D023|BR44_MOUSE & 0.9969 & 3 & 2 & YSLVIIPK \\
\hline sp|Q8K1A6|C2D1A_MOUSE & 0.9967 & 2 & 2 & SFDPVLEALSR \\
\hline sp|Q9CPR1|RWDD4_MOUSE & 0.9966 & 2 & 2 & SIYEGDNSFR \\
\hline sp|Q3TBW2|RM10_MOUSE & 0.996 & 2 & 2 & VFPSQVLKPFLENSK \\
\hline sp|Q8BK75|CC075_MOUSE & 0.9958 & 2 & 2 & GQLVFLEGLK \\
\hline sp|P50096|IMDH1_MOUSE & 0.9954 & 1 & 2 & NLIDAGVDGLR \\
\hline sp|P63139|NFYB_MOUSE & 0.9951 & 2 & 2 & EQDIYLPIANVAR \\
\hline sp|Q9R059|FHL3_MOUSE & 0.9949 & 2 & 2 & TLTQGGVTYR \\
\hline sp|Q3ULF4|SPG7_MOUSE & 0.9944 & 2 & 2 & EGGFSAFNQLK \\
\hline sp|Q80UZ2|SDA1_MOUSE & 0.9943 & 1 & 2 & DLLVQYATGK \\
\hline sp|Q8VE19|MIO_MOUSE & 0.9932 & 2 & 2 & NLAIFDLR \\
\hline sp|Q9JKK8|ATR_MOUSE & 0.9931 & 3 & 2 & FLDLIPQDTLAVASFR \\
\hline sp|Q8CIG3|AOF1_MOUSE & 0.9927 & 2 & 2 & VLVTVPLAILQR \\
\hline sp|Q3TDD9|KLRAQ_MOUSE & 0.9917 & 2 & 2 & EGLAQQVQQSLEK \\
\hline sp|Q6PCM2|INT6_MOUSE & 0.9917 & 1 & 2 & NLQAEGLTTLGQSLR \\
\hline sp|Q8K2F0|BRD3_MOUSE & 0.9917 & 1 & 2 & EYPDAQGFAADIR \\
\hline sp|Q8R0J7|VP37B_MOUSE & 0.9917 & 1 & 2 & SLAEGNLLYQPQLDAQK \\
\hline sp|Q9QUG2|POLK_MOUSE & 0.9912 & 2 & 2 & ASTVPAAISTAEEIFAIAK \\
\hline sp|Q68EF0|RAB3I_MOUSE & 0.9908 & 1 & 2 & IDVLQAEVAALK \\
\hline sp|Q7TPM1|BAT2L_MOUSE & 0.99 & 1 & 2 & LLSFSPEEFPTLK \\
\hline sp|O35379|MRP1_MOUSE & 1 & 2 & 2 & LYAWELAFQDK \\
\hline sp|P70399|TP53B_MOUSE & 1 & 3 & 2 & VITDVYYVDGTEVER \\
\hline sp|Q4FZC9|SYNE3_MOUSE & 1 & 2 & 2 & AATLLEQVTSSVR \\
\hline sp|Q6P8M1|TATD1_MOUSE & 1 & 2 & 2 & HQDDLQDVIER \\
\hline sp|Q8BK03|FA73B_MOUSE & 1 & 2 & 2 & PAAAYEEALQLVK \\
\hline
\end{tabular}




\begin{tabular}{|c|c|c|c|c|}
\hline sp|Q8BW10|NOB1_MOUSE & 1 & 2 & 2 & TDVFAPDYIAGVSPFAENDISSR \\
\hline sp|Q99K74|MED24_MOUSE & 1 & 2 & 2 & VESLVALLNNSSEM[147]K \\
\hline sp|Q9CQF4|CF203_MOUSE & 1 & 2 & 2 & SEQEEELESEPGVAK \\
\hline sp|Q9Z2A7|DGAT1_MOUSE & 1 & 2 & 2 & LQDSLFSSDSGFSNYR \\
\hline sp|P59016|VP33B_MOUSE & 0.9999 & 2 & 2 & AGLLTEQAPGDTLTAVESK \\
\hline sp|Q5DU25|IQEC2_MOUSE & 0.9999 & 2 & 2 & LIEAFSQR \\
\hline sp|Q8VDD9|PHIP_MOUSE & 0.9999 & 2 & 3 & YHDM[147]PDVIDFLVLR \\
\hline sp|A2AR02|PPIG_MOUSE & 0.9998 & 2 & 2 & DFM[147]VQGGDFSEGNGR \\
\hline sp|Q8C3X8|LMF2_MOUSE & 0.9998 & 2 & 2 & LFGSVEHLQLANSYGLFR \\
\hline sp|P70188|KIFA3_MOUSE & 0.9997 & 2 & 2 & SLNANTDITSLAR \\
\hline sp|Q9ER69|FL2D_MOUSE & 0.9997 & 2 & 2 & STM[147]VDPAINLFFLK \\
\hline sp|Q9QZ73|DCNL1_MOUSE & 0.9997 & 2 & 2 & QFM[147]IFTQSSEK \\
\hline sp|P58501|GCFC_MOUSE & 0.9996 & 2 & 2 & TLQELSIDGLLNR \\
\hline sp|P22366|MYD88_MOUSE & 0.9991 & 2 & 2 & FALSLSPGVQQK \\
\hline sp|P97480|EYA3_MOUSE & 0.999 & 2 & 2 & SNVGGLLSPQR \\
\hline sp|Q0P678|ZCH18_MOUSE & 0.999 & 2 & 3 & ASQQAAAPQPAVPGQPQQGSFVAHK \\
\hline sp|Q9CX00|K0174_MOUSE & 0.999 & 2 & 2 & ELDSGLAESVSTLIWAAPR \\
\hline sp|Q61037|TSC2_MOUSE & 0.9987 & 3 & 2 & LGYLPYSLLFR \\
\hline sp|P46938|YAP1_MOUSE & 0.9985 & 2 & 2 & GDSETDLEALFNAVM[147]NPK \\
\hline sp|P62046|LRCH1_MOUSE & 0.9985 & 2 & 2 & NLESIDPQFTIR \\
\hline sp|P97473|TRBP2_MOUSE & 0.9985 & 2 & 2 & TPISLLQEYGTR \\
\hline sp|Q5PSV9|MDC1_MOUSE & 0.9985 & 3 & 2 & LGLPLLSPEFLLTGVLK \\
\hline sp|Q9JKL4|CC060_MOUSE & 0.9985 & 2 & 2 & IEIVVVGTGNK \\
\hline sp|P97300|NPTN_MOUSE & 0.9984 & 2 & 3 & KRPDEVPDDDEPAGPM[147]K \\
\hline sp|Q99KY4|GAK_MOUSE & 0.9984 & 2 & 2 & IAVM[147]SFPAEGVESAIK \\
\hline sp|P59672|ANS1A_MOUSE & 0.9981 & 3 & 2 & LLLNGFDDVR \\
\hline sp|Q8VDR9|DOCK6_MOUSE & 0.9979 & 2 & 2 & VAELYLPLLSLAR \\
\hline sp|Q80VQ1|LRRC1_MOUSE & 0.9967 & 1 & 2 & SLEELLLDANQLR \\
\hline sp|Q9CXF7|CHD1L_MOUSE & 0.9963 & 2 & 2 & GIPTYIYYFPR \\
\hline sp|Q9D820|U566_MOUSE & 0.9962 & 2 & 2 & FVLDSAFLEGGHEK \\
\hline sp|Q8K2J4|CCD14_MOUSE & 0.9952 & 3 & 2 & NVSQTAEK \\
\hline sp|P49769|PSN1_MOUSE & 0.9937 & 2 & 2 & NETLFPALIYSST \\
\hline sp|Q3V1H1|CKAP2_MOUSE & 0.9924 & 2 & 2 & IEPITSPIENIISIYEK \\
\hline sp|P70236|MP2K6_MOUSE & 0.9923 & 2 & 2 & ADDLEPIVELGR \\
\hline sp|O35375|NRP2_MOUSE & 0.9917 & 1 & 2 & IFQANNDATEVVLNK \\
\hline sp|P50427|STS_MOUSE & 0.9917 & 1 & 2 & VLAALDELGLAR \\
\hline sp|P84089|ERH_MOUSE & 0.9917 & 1 & 2 & ADTQTYQPYNK \\
\hline sp|Q5DTK1|CHSS3_MOUSE & 0.9917 & 1 & 2 & DNTVQGQQVYYPIIFSQYDPK \\
\hline sp|Q8K4R9|DLGP5_MOUSE & 0.9917 & 1 & 2 & ANEILVQQGLESLTDR \\
\hline sp|Q91YU8|SSF1_MOUSE & 0.9917 & 1 & 2 & TEEELQAILAAK \\
\hline sp|Q9CQ39|MED21_MOUSE & 0.9917 & 1 & 2 & IQSALADIAQSQLK \\
\hline sp|Q9Z0R4|ITSN1_MOUSE & 0.9917 & 1 & 2 & LQEIDVFNNQLK \\
\hline sp|Q8VCF0|MAVS_MOUSE & 0.9914 & 2 & 2 & DTLWGLFNNLQR \\
\hline sp|Q6TEK5|VKORL_MOUSE & 0.9908 & 1 & 2 & GFGLLGSIFGK \\
\hline sp|Q9JIA7|SPHK2_MOUSE & 0.9908 & 1 & 2 & LLILVNPFGGR \\
\hline
\end{tabular}




\begin{tabular}{|c|c|c|c|c|}
\hline sp|Q99LC9|PEX6_MOUSE & 0.9902 & 3 & 2 & LVFVGASEDR \\
\hline sp|Q3U1G5|I20L2_MOUSE & 0.9985 & 2 & 2 & IDLLGEFQSALPK \\
\hline sp|Q8BXA1|GOLI4_MOUSE & 0.9981 & 2 & 2 & DAGFQALEEQNQVEPR \\
\hline sp|P70158|ASM3A_MOUSE & 0.998 & 2 & 2 & NGNPLNSVFVAPAVTPVK \\
\hline sp|Q61624|ZN148_MOUSE & 0.9979 & 2 & 2 & LPQGLQYALNVPISVK \\
\hline sp|Q9QXE7|TBLX_MOUSE & 0.9979 & 2 & 2 & HQEPVYSVAFSPDGK \\
\hline sp|P59997|JHD1A_MOUSE & 0.9974 & 2 & 2 & ILLEELASSDPK \\
\hline sp|Q8BRG8|TM209_MOUSE & 0.9918 & 2 & 2 & YTVAPTSLVVSPGQQALLGLK \\
\hline sp|Q3URD3|SLMAP_MOUSE & 0.9917 & 1 & 2 & IEALQADNDFTNER \\
\hline sp|Q62388|ATM_MOUSE & 0.9917 & 1 & 2 & SVATSSIVGYILGLGDR \\
\hline sp|Q7TMW6|NARFL_MOUSE & 0.9917 & 1 & 2 & APDTEGSELLQQLER \\
\hline sp|Q80YR4|ZN598_MOUSE & 0.9917 & 1 & 2 & TPGLAPTPQAYLVPENFR \\
\hline sp|Q8K3K8|OPTN_MOUSE & 0.9917 & 1 & 2 & ADLLGIVSELQLK \\
\hline sp|Q9CPS7|PNO1_MOUSE & 0.9917 & 1 & 2 & RPVFPPLSGDQLLTGK \\
\hline sp|P70261|PALD_MOUSE & 0.9908 & 1 & 2 & ALGNILAYLSDAK \\
\hline sp|Q8BW49|TTC12_MOUSE & 0.9908 & 1 & 2 & ANTAIGILTDLALEER \\
\hline sp|Q91W92|BORG5_MOUSE & 0.9908 & 1 & 2 & LTADM[147]ISPPLGDFR \\
\hline sp|Q9Z0P7|SUFU_MOUSE & 0.9908 & 1 & 2 & VSILPDVVFDSPLH \\
\hline sp|P62737|ACTA_MOUSE & 1 & 5 & 2 & AVFPSIVGR \\
\hline sp|P12246|SAMP_MOUSE & 0.9965 & 1 & 2 & APPSIVLGQEQDNYGGGFQR \\
\hline sp|P62835|RAP1A_MOUSE & 0.9952 & 1 & 2 & INVNEIFYDLVR \\
\hline sp|Q80Y17|L2GL1_MOUSE & 0.9948 & 1 & 2 & APVVAIAVLDGR \\
\hline
\end{tabular}

Table S3

\begin{tabular}{|c|c|}
\hline \multicolumn{2}{|c|}{ All the abbreviated words and their full name } \\
\hline 1DE-LC-MS/MS & dimensional gel electrophoresis- liquid chromatography-mass spectrometry \\
\hline $\mathrm{ACN}$ & Acetonitrile \\
\hline BSA & Bovine Albumin Standards \\
\hline DMEM & Dulbecco's Modified Eagle Media \\
\hline Dnmt3a & DNA methyltransferase $3 \mathrm{~A}$ \\
\hline Dnmt3a-D & Dnmt3a depletion B16 melanoma \\
\hline ERK & extracellular signal-regulated kinase \\
\hline FBS & Fetal bovine serum \\
\hline GO & Genome Ontology \\
\hline IEF & isoelectro- focusing \\
\hline IPG & immobilized $\mathrm{pH}$ gradient \\
\hline IPI & International Protein Index \\
\hline JNK & c-Jun N-terminal kinase \\
\hline KEGG & Kyoto Encyclopedia of Genes and Genomes \\
\hline LTQ-Orbitrap & Linear ion trap Orbitrap \\
\hline MAPK & mitogen activated protein kinase signaling pathway \\
\hline $\mathrm{NC}$ & negative control cell line \\
\hline PBS & phosphate-buffered saline \\
\hline PMSF & phenylmethanesulfonyl fluoride \\
\hline TPP & Trans-Proteomic Pipeline \\
\hline
\end{tabular}

